\title{
Loss of ACKR4 in tumor cells dysregulates dendritic cell migration to tumor-draining lymph nodes and T-cell priming
}

Dechen Wangmo ${ }^{1}$, Prem K. Premsrirut ${ }^{2}$, Ce Yuan ${ }^{1}$, William S. Morris ${ }^{1}$, Xianda Zhao ${ }^{1 *}$, and Subbaya Subramanian ${ }^{1,3,4^{*}}$

${ }^{1}$ Department of Surgery, University of Minnesota Medical School, Minneapolis, MN, 55455, USA ${ }^{2}$ Miramus Inc, Brooklyn, NY, 11226, USA

${ }^{3}$ Masonic Cancer Center, University of Minnesota, Minneapolis, MN 55455, USA

${ }^{4}$ Center for Immunology, University of Minnesota, Minneapolis, MN 55455, USA

Running title: ACKR4 regulates dendritic cell migration and T-cell priming

\section{${ }^{*}$ Correspondence}

Subbaya Subramanian, MS, PhD

Xianda Zhao MD, PhD

Department of Surgery

University of Minnesota Medical School

11-212 Moos Tower

Mayo Mail Code 195

420 Delaware Street SE

Minneapolis, MN 55455

Tel: 612-626-4330

Email: subree@umn.edu

Conflicts of interest: No conflicts of interest to declare 


\section{LAY SUMMARY}

Our study demonstrated that Atypical Chemokine Receptor 4 (ACKR4) is downregulated in human colorectal cancer (CRC) tissues compared with normal colon tissues. Loss of ACKR4 in human $\mathrm{CRC}$ tissues is associated with a weak anti-tumor immune response. Knockdown of ACKR4 in tumor cells impairs the dendritic cell migration from the tumor tissue to the tumordraining lymph nodes (TdLNs), causing inadequate tumor-specific T-cell expanding and insensitivity to immune checkpoint blockades. However, loss of ACKR4 in tumor stromal cells does not significantly affect anti-tumor immunity. In human CRC tumors, high expression of microRNA-552 is a mechanism leading to ACKR4 downregulation. Our study revealed a novel mechanism that leads to the poor immune response in a subset of CRC tumors and will contribute to the framework for identifying new therapies against this deadly tumor. 


\section{ABSTRACT}

Colorectal cancer $(\mathrm{CRC})$ is one of the most common malignancies in both morbidity and mortality. Immune checkpoint blockade (ICB) treatments have been successful in a portion of mismatch repair-deficient (dMMR) CRC patients but failed in the mismatch repair-proficient (pMMR) CRC patients. Atypical Chemokine Receptor 4 (ACKR4) is known for regulating dendritic cell (DC) migration. However, the roles of ACKR4 in CRC development and immunoregulation are unclear. By analyzing human CRC tissues, transgenic animals, and genetically modified CRC cells lines, our study revealed an important function of ACKR4 in maintaining $C R C$ immune response. Loss of ACKR4 in CRC is associated with poor immune infiltration in the tumor microenvironment. More importantly, loss of ACKR4 in CRC tumor cells, rather than stromal cells, restrains the DC migration and antigen presentation to the tumordraining lymph nodes (TdLNs). Tumors with ACKR4 knockdown become less sensitive to immune checkpoint blockades. Finally, we identified that microRNA-552 negatively regulates ACKR4 expression in human CRC. Taken together, our work identifies a critical mechanism for the maintenance of the DC-mediated T-cell priming in the TdLNs. These new findings demonstrate a novel mechanism leading to immunosuppression and ICB treatment resistance in CRC tumors.

Keywords: Colorectal Cancer, Immune Checkpoints, Dendritic Cells, Atypical Chemokine Receptor 4 (ACKR4), T-cell priming, Immune checkpoint blockade 


\section{INTRODUCTION}

Colorectal cancer $(\mathrm{CRC})$ is the third most commonly diagnosed malignancy and the third leading cause of cancer-related deaths in the United States[1]. By 2030, the global CRC burden is expected to increase by $60 \%$ and surpass 2.2 million new cases and 1.1 million deaths[2]. The paradigm shift in cancer treatment brought by immunotherapy has been a major scientific and clinical breakthrough. Since the first immune checkpoint blockades (ICB) approval for melanoma, ICB has developed itself as the standard of care for multiple types of cancers, including the mismatch repair-deficient (dMMR)/microsatellite instability-high (MSI-H) CRC tumors[3]. However, not all dMMR/MSI-H CRC tumors are sensitive to ICB, and all of the mismatch repair-proficient (pMMR)/microsatellite instability-low (MSI-L)/microsatellite stability (MSS) CRC tumors are resistant to ICB[4]. Understanding the mechanisms of immunosuppression and immune therapy resistance is, therefore, critical for designing novel treatments for CRC patients.

The immunogenicity of tumors is fundamental for ICB treatment. Poorly immunogenic tumors present a hallmark feature of sparse tumor T-cell infiltration[5]. One of the key mechanisms involved in poor T-cell infiltration has been attributed to defects in the antigen presentation process, which significantly weakens the tumor-specific T-cell priming and precludes theT-cell mediated killing of cancer cells[6]. Dendritic cells (DCs) are the most potent antigen-presenting cells necessary to prime and activate tumor-antigen specific T cells to induce an effective antitumor immune response[7]. Previous studies have shown that dysfunction of DCs caused defective antigen presentation and T-cell priming, leading to uncontrolled tumor development and ICB resistance in multiple cancers[8-10].

Successful antigen presentation by the DCs involves efficient migration of DCs from the tumor tissue to the regional lymph nodes. DC migration heavily depends on CCR7, a G-protein coupled receptor for two chemokines: CCL19 and CCL21[5,11,12]. CCL21 has an extended 
positively charged $\mathrm{C}$ terminus that limits its interstitial diffusion, causing a stable gradient of CCL21 that directs the CCR7 expressing DCs from the tissue interstitium into lymphatic vessels[12,13]. On the other hand, both CCL19/21 are ligands for the atypical chemokine receptor 4 (ACKR4), a scavenging receptor that internalizes and mediates lysosomal degradation of CCL19/21[14]. It is known that ACKR4 controls the bioavailability of CCL19/21, creating a CCL19/21 gradient that facilitates the directional migration of DCs from the nonlymphatic tissue to the draining lymph node[5,12-14]. However, the effects of ACKR4 in CRC progression and immunoregulation are largely unknown. Here, we examined the function of ACKR4 in CRC tumor progression and anti-itumor immunity, emphasizing its role in the DCsmediated antigen presentation process and subsequent $\mathrm{T}$ cell activation. Our study provides deeper insights into the immunoregulation in CRC and potentially leads to novel approaches for maximizing $C R C$ response to ICB.

\section{MATERIAL AND METHODS}

\section{Cell lines and organoids}

Human CRC cell lines SW480, HT29, HT116, DLD-1, WiDr, Caco-2, and HCT-8, and murine CRC cell line MC38 were used in the study. Human primary organoids were also used. The detailed information regarding cell culture and organoids culture was included in our previous publication[15].

\section{Immunofluorescence and Histology}

Human CRC tumor tissues were fixed in $10 \%$ formalin before paraffin embedding. Sections of formalin-fixed paraffin-embedded (FFPE) tissues were deparaffinized with xylene and rehydrated with ethanol (twice in 100\%, 90\%, $80 \%$, and $70 \%$ ). The sections were heated in a boiling water bath with citric buffer for 12 minutes to retrieve antigens. Next, the sections were 
blocked by incubating for 30 minutes in $5 \%$ bovine serum albumin buffer. Tissues were incubated overnight at $4{ }^{\circ} \mathrm{C}$ with primary antibodies: anti-ACKR4 antibody (Novus), anti-CD3 (Abcam), and anti-CD11c (Abcam). The next day, the sections were washed and incubated with fluorescence-conjugated secondary antibodies (1:1000 dilution, ThermoFisher) at room temperature for 1 hour. After washing, the slides were mounted with ProLong Gold antifade mountant with DAPI and imaged. The information of primary antibodies was included in Supplementary Table 1.

\section{Western blotting of ACKR4}

Total protein of $40 \mu \mathrm{g}$ was prepared from each sample and quantified by the Pierce ${ }^{\mathrm{TM}} \mathrm{BCA}$ Protein Assay Kit (ThermoFisher). We ran the protein in sodium dodecyl sulfate-polyacrylamide (SDS) gel electrophoresis. The proteins from the gel were transferred to polyvinylidene difluoride membranes (ThermoFisher), blocked with 5\% BSA, and incubated in primary antibodies overnight at $4{ }^{\circ} \mathrm{C}$. The primary antibodies are anti-ACKR4 (Abcam) and anti-betaactin (Cell Signaling). The next day, the membranes were washed and incubated in peroxidaselinked anti-rabbit IgG and peroxidase-linked anti-mouse $\lg G$ for 1 hour at room temperature. Pierce $^{\mathrm{TM}}$ ECL western blotting substrate (ThermoFisher) was used to image the membranes.

\section{Cell line transfection and transduction}

We used the ACKR4 shRNA expressing lentiviral vectors to knock down ACKR4 expression in the MC38 cell line. Briefly, $5 \mu \mathrm{g}$ of DNA $(2.5 \mu \mathrm{g}$ of mixed shRNA expressing plasmids and 2.5 $\mu \mathrm{g}$ of pPACKH1-XL packaging vector) was mixed with $10 \mu \mathrm{L} \mathrm{P3000}{ }^{\mathrm{TM}}$ reagent in $250 \mu \mathrm{L}$ OptiMEM medium. Then the diluted DNA was added to $250 \mu \mathrm{L}$ Lipofectamine $^{\mathrm{TM}} 3000$ Transfection Reagent and incubated for 15 minutes at room temperature. The mixture was added to $5^{\star} 10^{5}$ HEK293TN cells in one well of a 6 -well plate. Another $500 \mu \mathrm{L}$ Opti-MEM medium was added to make the final volume of $1000 \mu \mathrm{L} .24$ hours after the transfection, we changed the Opti-MEM 
medium to normal cell growth media and cultured the cells for another 24 hours. Then the viruscontaining media was collected and added to wild-type MC38 at different titrations. Empty shRNA vectors served as the negative control. Three days after the transduction, the transduced MC38 cells were subjected to antibiotic selection. After one week of antibiotic selection, we performed a western blotting analysis of ACKR4 to validate the knockdown.

\section{Dendritic cell isolation}

Dynabeads Untouched Mouse DC Enrichment Kit (ThermoFisher) was used, and manufacturers' instruction was followed. Briefly, murine PBMCs were isolated from spleen, bilateral inguinal, brachial, and axillary lymph nodes by gradient centrifugation. The cells were incubated in antibody mix for 20 mins at $2^{\circ} \mathrm{C}$ to $8^{\circ} \mathrm{C}$, washed, and then incubated with Depletion MyOne SA Dynabeads magnetic beads for 15 mins at $2^{\circ} \mathrm{C}$ to $8^{\circ} \mathrm{C}$. The tube was placed in the magnet, and the untouched DCs in the supernatant were cultured in Iscove's Modified Dulbecco's Medium (IMDM) supplemented with 2,000 IU/mL IL4, 2,000 IU/mL granulocyte-macrophage colony-stimulating factor, and 2,000 IU/mL tumor necrosis factor.

\section{In vivo DCs migration assay}

We resuspended $3^{*} 10^{5}$ million freshly enriched CD $45.1^{+}$DCs in $50 \mu \mathrm{PBS}$. We then injected them into multiple sites of MC38 subcutaneous tumors growing in C57BL/6 mouse with different ACKR4 expression ( $500 \mathrm{~mm}^{3}, 3^{*} 10^{5}$ million/tumor) by a syringe with a $30 \mathrm{G}$ needle. Thirty-six hours after the injection, we sampled the tumor-draining lymph nodes (the unilateral inguinal and axillary lymph nodes). Then we isolated single cells from the tumor-draining lymph nodes (TdLNs) for detecting CD45.1 $1^{+}$DCs by FACS analysis.

\section{Flow cytometry}

Mouse tumor tissues were minced into small pieces $\left(2^{\star} 2 \mathrm{~mm}\right)$ and digested with collagenase IV $(0.5 \mathrm{mg} / \mathrm{mL})$ and deoxyribonuclease $(50$ units $/ \mathrm{mL})$ for 1 hour at $37^{\circ} \mathrm{C}$. The digested tumor 
tissues and lymphatic tissues (TdLNs and spleens) were meshed and flushed through $70 \mu \mathrm{M}$ strainer and $40 \mu \mathrm{M}$ strainer, respectively. Red blood cells were lysed by incubating the cells with red blood cell lysis buffer for 15 minutes and neutralizing with PBS. The cells were counted using a hemacytometer. Zombie Green fixable viability dye (BioLegend) was used to count live and dead cells. All the cells were stained with primary antibody cocktails for cell surface markers. For cytoplasmic staining, cells were treated with the Cyto-Fast Fix-Perm Buffer set (BioLegend).

All samples were fixed after staining. The samples were immediately analyzed in a BD FACSCanto (BD Biosciences) cytometry to prevent signal deterioration. All the data were analyzed with the FlowJo. The information of primary antibodies was included in Supplementary Table 1.

\section{Antigen presentation assay}

We cultured $3^{*} 10^{5}$ million freshly enriched DCs in $2 \mathrm{ml}$ Dendritic Cell Base Media (R\&D systems) plus $10 \%$ FBS. $40 \mu$ g of ovalbumin (OVA) peptides (257-264, AnaSpec) were supplied to the DCs culture for a final concentration of $20 \mu \mathrm{g} / \mathrm{ml}$. After 18 hours of DCs pulsing, we collected the DCs and injected them into multiple sites of MC38 subcutaneous tumors growing in C57BL/6 mouse with different ACKR4 expression ( $300 \mathrm{~mm}^{3}, 3^{*} 10^{5}$ million/tumor) by a syringe with a $30 \mathrm{G}$ needle. Two weeks later, we collected the TdLNs for OVA-specific T-cell analysis.

Single cells were isolated from the TdLNs by mechanical tissue dissociation. Then, $3^{\star} 10^{5}$ million single cells were resuspended in $1000 \mu$ PBS with cell $1 \mu$ Z Zombie Green Fixable Viability dye and incubated at room temperature for 15 minutes. After washing, the cells were blocked with TruStain FcX'TM PLUS (0.25 $\mu \mathrm{g}$, Biolegend) and stained with Tetramer/BV421 - H-2 Kb OVA

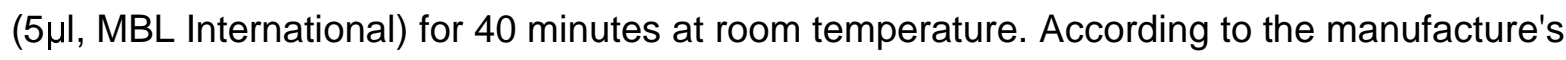
instruction and our preliminary experiment optimization, we used an anti-CD8 (clone KT15) antibody (MBL International) to minimize the false-positive rate of the tetramer staining. Lymphatic cells from naïve mice were used as a negative control. 


\section{Mouse subcutaneous models}

The subcutaneous model was established by resuspending $5^{\star} 10^{5} \mathrm{MC} 38$ cells in $100 \mu \mathrm{L}$ Matrigel (BD Bioscience) and injecting the tumor cell suspension into the right flank of naïve C57BL/6 mice. Following injection, using an electronic caliper, tumor growth was monitored and measured 1-2 times a week. Tumor volume was calculated using the formula (length*width²)/2.

\section{Mouse treatment}

Mice were treated with either $\mathrm{lgG}(5 \mathrm{mg} / \mathrm{kg}$ as an anti-4-1BB control, $10 \mathrm{mg} / \mathrm{kg}$ as an anti-PD-1 control, BioXcell), anti-4-1BB agonist (5 mg/kg, clone: 3H3, BioXcell), or anti-PD-1 (10 mg/kg, clone: RMP1-14, BioXcell) on day 10, 14, and 18. All treatments were given intraperitoneally.

\section{qPCR analysis}

The mirVana microRNA (miRNA) Isolation Kit (Thermo Fisher Scientific) was used to extract total RNA from tumor cell lines and tissues. $500 \mathrm{ng}$ of total RNA was used for establishing the cDNA library with the miScript II RT Kit (Qiagen). qRT-PCR was performed with the SYBR Green I Master kit (Roche Applied Science) in a LightCycler 480. The following forward primers were used: miR-552: GTTTAACCTTTTGCCTGTTGG and U6 snRNA:

AAGGATGACACGCAAATTCG. The RT kit provides the universal reverse primer.

\section{Enzyme-Linked Immunosorbent Assay (ELISA) for CCL21}

CCL21 was quantified in tumor tissues and tumor-draining lymph nodes using an ELISA kit (Abcam). Briefly, tissue lysate samples were prepared by homogenizing tumor tissues and tumor-draining lymph nodes. We normalized the protein concentration between different samples before loading them to the experiment. The manufacturer's instructions were followed every step. 
Statistical analysis

We performed all statistical analyses and graphing using GraphPad Prism software (Version 8). Data were displayed as means \pm SEMs. For comparison of two groups' quantitative data, paired or unpaired Student's t-test was used. For multiple groups' comparison, one-way analysis of variance (ANOVA) was used followed by Bonferroni correction. Kaplan-Meier curves and logrank tests were used to compare survival outcomes between groups. We used the chi-square test to compare two variables in a contingency table to see if they are related. A two-tail P-value of less than 0.05 was considered statistically significant.

\section{RESULTS}

\section{ACKR4 is downregulated in CRC compared with normal colon}

To investigate the immunoregulatory role of ACKR4 in CRC, we first evaluated the ACKR4 expression in CRC tissues and normal colon tissues as the control. Analysis of the CRC dataset in the TCGA project and another independent project reported by Vasaikar et al.[16] showed that ACKR4 expression is lower in CRC tissues than in normal colon tissues (Figure 1A-B). Further stratification of the CRC cases based on the MSI/MSS statuses indicated that ACKR4 expression is lower in MSS/MSI-L tumors than the MSI-H tumors (Figure $1 \mathrm{~A}-\mathrm{B}$ ). The immunofluorescence on sections of 68 human colorectal tumors and 17 normal colons revealed that $88 \%$ of normal colon tissues and $78 \%$ of MSI-CRC tissues have abundant ACKR4 expression. In contrast, only $45 \%$ of MSS-CRC tissues have a similar ACKR4 level. These data confirmed the downregulation of ACKR4 in CRC tissues, especially in the MSS subtype cases (Figure 1C). Next, we evaluated the prognostic value of ACKR4 in the TCGA cohort (Figure 1D). Although not statistically significant, patients with higher ACKR4 expression are more likely to have a longer median survival time than patients with lower ACKR4 expression (Figure 1D). 
Finally, we screened the ACKR4 level in different human and mouse CRC cell lines by westernblot analysis (Figure 1E). Notably, the mouse CRC cell line MC38 (MSI phenotype) has significantly higher ACKR4 expression than the CT26 cell line (MSS phenotype).

\section{Knockdown of ACKR4 in tumor cells but not the host tissues accelerate tumor growth}

Next, we sought to understand the impact of ACKR4 downregulation in CRC development. Using the vector-based short hairpin RNA (shRNA) interference technology, we knocked down ACKR4 expression in the MC38 cell line, which has relatively high endogenous ACKR4 expression (Figure 2A and Figure 1E). Knockdown of ACKR4 did not significantly influence the MC38 cell proliferation in vitro (Figure $2 \mathrm{~A}$ ). We then inoculated the MC38 cells subcutaneously into naïve C57BL/6 mice. Notably, the knockdown of ACKR4 in the tumor cells accelerated tumor growth in vivo (Figure 2B). To see whether the ACKR4 level in the host tissue also affects tumor development, we established a conditional ACKR4 knockdown mouse model (Figure 2C). We knocked down ACKR4 expression in the host mice by doxycycline treatment right before MC38 tumor cell injection. However, the knockdown of ACKR4 in host tissue did not significantly alter the tumor development (Figure 2D). Our results indicated that ACKR4 of tumor cells is more competent in regulating tumor growth than the host ACKR4.

\section{Loss of ACKR4 reduces tumor T-cell infiltration}

To study whether the tumor growth caused by ACKR4 knockdown is associated with anti-tumor immunity, we analyzed the tumor immune infiltration in the TCGA CRC data set by the CIBERSORT algorithm (Figure 3A and B). Tumors with higher ACKR4 expression have more immune cell infiltration, including the T-cell and DCs, than tumors with lower ACKR4 expression (Figure 3B). Our histological analysis on human CRC tumors confirmed that ACKR4 highexpressing tumors are associated with a higher amount of tumor-infiltrating T-cell (Figure 3C). However, there is no difference in DCs infiltration between the ACKR4-high and -low groups 
(Figure 3C). Next, we investigated the immune infiltration in ACKR4 knockdown tumor models (Figure S1A and B). Our results show that ACKR4 knockdown tumors have fewer CD4 ${ }^{+}$T cells but a higher proportion of exhausted $\mathrm{CD} 4^{+} \mathrm{T}$ cells in their tumor microenvironment than the control group (Figure 3D). However, the frequencies of tumor-infiltrating $\mathrm{CD}^{+} \mathrm{T}^{\mathrm{T}}$ cells and DCs are not influenced by ACKR4 expression (Figure 3D and Figure S1C). The ACKR4 level in tumor cells also does not systemically change the frequency and function of immune cells in the tumor-draining lymph nodes (Figure S1C).

\section{Loss of ACKR4 impairs dendritic cell migration to tumor-draining lymph nodes and tumor-specific T-cell expansion}

Since ACKR4 regulates the CCL21 chemokine gradient[12], we hypothesize that loss of ACKR4 in tumor tissue will increase the CCL21 amount in the tumor microenvironment. An increase of CCL21 in the tumor tissue will potentially impede the DCs migration, mediated by the CCL21 chemokine gradient between the tumor tissue and the tumor-draining lymph nodes (TdLNs). To validate this hypothesis, we injected the CD $45.1^{+}$DCs into tumors with wild-type or knockeddown ACKR4 expression. We then analyzed the amount of CD45.1 $1^{+}$Cs in the TdLNs. Notably, DCs in the wild-type and control tumors are more likely to migrate to the TdLNs than the DCs in the ACKR4 knockdown tumors (Figure 4A). To see whether the reduction of DCs migration will cause the impaired tumor-specific T-cell priming in the TdLNs, we tested for the antigen-specific T cells in the TdLNs. We first pulsed the DCs with the Ovalbumin (OVA) antigen and then injected them into the tumors. We analyzed the OVA-specific CD8 ${ }^{+} \mathrm{T}$ cells in the TdLNs and found that AKCR4 knockdown in the tumor significantly reduced the DCs mediated antigenspecific T-cell priming in the TdLNs (Figure 4B). We also confirmed the finding with the endogenous tumor antigen (Figure 4C). Finally, we determined that the CCL21 level in the ACKR4 knockdown tumor tissues is significantly higher than in the wild-type and control groups (Figure 4D). 


\section{Loss of ACKR4 weakens tumor response to immune checkpoint blockades}

Because ACKR4 knockdown reduces the T-cell tumor infiltration and DCs mediated tumorspecific T-cell expansion in the TdLNs (Figure 3 and 4), we next evaluated whether ACKR4 knockdown affects the tumor response to immune checkpoint blockades. We treated the wildtype, control, and ACKR4 knockdown tumors with anti-PD-1 or anti-4-1BB antibodies. The ACKR4 knockdown tumors are less sensitive to anti-PD-1 or anti-4-1BB treatments than wildtype and control tumors (Figure 5). This result suggested that loss of ACKR4 involves the immune checkpoint blockades resistance in colorectal cancers.

\section{microRNA miR-552 downregulates ACKR4 in CRC tumors}

Our previous microRNA (miRNA) expression profiling analysis has shown that miR-552 is highly expressed in the MSS CRC tumors, which do not respond to immune checkpoint blockades[17]. Further sequence match analysis showed that miR-552 potentially binds to ACKR4 transcript and subsequently downregulates ACKR4 expression (Figure 6A). Our dual luciferase assay and flow cytometry analysis confirmed the effects of miR-552 on ACKR4 downregulation in human CRC cell lines (Figure 6A and B). In primary human CRC tissues, the ACKR4 protein expression level is negatively correlated with the miR-552 expression level (Figure 6C). Analysis of the TCGA CRC dataset further confirmed the negative correlation between miR-552 and ACKR4 (Figure 6D).

\section{DISCUSSION}

Investigating the regulatory mechanism of tumor immunity is essential to alleviate drug resistance and improve the effect of immunotherapy[18]. As the most important cell type in the process of antigen presentation, DCs and their function are closely related to the intensity of tumor immunity[8-10]. The CCR7 expressed on DCs and the CCL19/21 gradient in the 
interstitial compartment largely controls the DCs migration[11,12]. ACKR4 shapes the CCL19/21 gradient between the non-lymphatic and lymphatic tissues by scavenging both the soluble and immobilized CCL19/CCL21[11,12]. In breast cancer, nasopharyngeal cancer, liver cancer, and cervical cancer, ACKR4 negatively regulates tumor growth and metastasis, implying a protective role in tumorigenesis[19-22]. However, the role of ACKR4 in tumor immunogenicity and overall anti-tumor immunity of $\mathrm{CRC}$ is unclear.

Our study first evaluated the expression of ACKR4 in normal human colon and colon cancer tissues and revealed that ACKR4 is downregulated in CRC tumors. This result echoes a recent study showing that villous colon adenomas have less ACKR4 expression than the normal colon tissues[23]. Further subgroup analysis indicated that the MSI-H CRC tumors have more ACKR4 expression than the MSI-L/MSS CRC tumors. These data established the association between ACKR4 expression and CRC development, providing the cornerstone for further studying the biological functions of ACKR4 in CRC.

A key question is whether AKCR4 of tumor cells or ACKR4 of tumor stromal cells affects tumor development. Taking advantage of inducible ACKR4 knockdown mice, we were able to allow the mice to mature with intact ACKR4 expression and selectively downregulate the ACKR4 expression in the host right before and during wild-type MC38 tumor development. In another model, we knocked down ACKR4 in MC38 cells, which have a relatively high endogenous ACKR4 expression, and inoculated those cells to wild-type mice. Notably, ACKR4 knockdown in MC38 tumor cells significantly accelerated tumor growth. However, ACKR4 expression on tumor stromal cells did not affect tumor growth. These results highlighted the distinct functions of ACKR4 in the tumor cell and the stromal compartments. Our data is different from the previously published paper showing that ACKR4 knockout mice delayed E0771 mammary tumor growth[5]. These differences may be due to different tumor cell lines tested. Although there is still controversy, permanent germline ACKR4 knockout may cause abnormalities in 
immune organ development[24-26]. This might be another reason why our results from inducible ACKR4 knockdown mice are different from embryonic ACKR4 knockout mice.

DCs have been identified as the most potent antigen-presenting cells in tumor antigen presentation and T-cell priming[7-10]. ACKR4, a decoy receptor that binds and degrades CCR7 ligands CCL19/CCL21, regulates DCs migration from skin to the regional lymph nodes[12]. However, whether similar effects exist in tumor conditions remains unknown. Our work proved that in the case of ACKR4 knockdown, tumor-infiltrating DCs are less likely to migrate towards TdLNs, causing a weak tumor-specific T-cell expansion in TdLNs. Consequently, the intensity of anti-tumor immunity and response to ICB is significantly restricted by ACKR4 loss. These data echos our previous work showing that the immune response that happens in TdLNs is extremely critical for initiating anti-tumor immunity[27]. In addition, our study also indicates that miR-552 negatively regulates ACKR4, and blocking the function of miR-552 increases ACKR4 expression in human $\mathrm{CRC}$ cell lines. Those results provided a potential target to rescue the ACKR4 expression in tumors.

In conclusion, our work indicated that loss of ACKR4 in CRC is associated with poor anti-tumor immune infiltration. Mechanistically, the knockdown of ACKR4 in tumor cells restricts the DCs migration from tumor tissue to the TdLNs, thus impairing the tumor-specific T-cell priming and response to ICB. These data, collectively, describe a novel immunosuppressive mechanism and increase our understanding of how intrinsic tumor factors affect DC-mediated immune response in CRC.

\section{Acknowledgments}

This study was supported by the Minnesota Colorectal Cancer Research Foundation and the National Institutes of Health grant R03CA219129. Dechen Wangmo was supported by the 
National Institutes of Health's National Center for Advancing Translational Sciences, grants TL1R002493 and UL1TR002494. The content is solely the responsibility of the authors and does not necessarily represent the official views of the National Institutes of Health's National Center for Advancing Translational Sciences.

\section{Reference}

1. Siegel, R.L.; Miller, K.D.; Fuchs, H.E.; Jemal, A. Cancer Statistics, 2021. CA: a cancer journal for clinicians 2021, 71, 7-33, doi:10.3322/caac.21654.

2. Siegel, R.L.; Torre, L.A.; Soerjomataram, I.; Hayes, R.B.; Bray, F.; Weber, T.K.; Jemal, A. Global patterns and trends in colorectal cancer incidence in young adults. Gut 2019, 68, 2179-2185, doi:10.1136/gutjnl-2019-319511.

3. Stein, A.; Moehler, M.; Trojan, J.; Goekkurt, E.; Vogel, A. Immuno-oncology in Gl tumours: Clinical evidence and emerging trials of PD-1/PD-L1 antagonists. Critical reviews in oncology/hematology 2018, 130, 13-26, doi:10.1016/j.critrevonc.2018.07.001.

4. Lumish, M.A.; Cercek, A. Immunotherapy for the treatment of colorectal cancer. Journal of surgical oncology 2021, 123, 760-774, doi:10.1002/jso.26357.

5. Whyte, C.E.; Osman, M.; Kara, E.E.; Abbott, C.; Foeng, J.; McKenzie, D.R.; Fenix, K.A.; Harata-Lee, Y.; Foyle, K.L.; Boyle, S.T., et al. ACKR4 restrains antitumor immunity by regulating CCL21. The Journal of experimental medicine 2020, 217, doi:10.1084/jem.20190634.

6. Wang, S.; He, Z.; Wang, X.; Li, H.; Liu, X.S. Antigen presentation and tumor immunogenicity in cancer immunotherapy response prediction. elife 2019, 8, doi:10.7554/eLife.49020.

7. Wang, Y.; Xiang, Y.; Xin, V.W.; Wang, X.W.; Peng, X.C.; Liu, X.Q.; Wang, D.; Li, N.; Cheng, J.T.; Lyv, Y.N., et al. Dendritic cell biology and its role in tumor immunotherapy. Journal of hematology \& oncology 2020, 13, 107, doi:10.1186/s13045-020-00939-6.

8. Roberts, E.W.; Broz, M.L.; Binnewies, M.; Headley, M.B.; Nelson, A.E.; Wolf, D.M.; Kaisho, T.; Bogunovic, D.; Bhardwaj, N.; Krummel, M.F. Critical Role for CD103(+)/CD141(+) Dendritic Cells Bearing CCR7 for Tumor Antigen Trafficking and Priming of T Cell Immunity in Melanoma. Cancer cell 2016, 30, 324-336, doi:10.1016/j.ccell.2016.06.003.

9. Salmon, H.; Idoyaga, J.; Rahman, A.; Leboeuf, M.; Remark, R.; Jordan, S.; Casanova-Acebes, M.; Khudoynazarova, M.; Agudo, J.; Tung, N., et al. Expansion and Activation of CD103(+) Dendritic Cell Progenitors at the Tumor Site Enhances Tumor Responses to Therapeutic PD-L1 and BRAF Inhibition. Immunity 2016, 44, 924-938, doi:10.1016/j.immuni.2016.03.012.

10. Binnewies, M.; Mujal, A.M.; Pollack, J.L.; Combes, A.J.; Hardison, E.A.; Barry, K.C.; Tsui, J.; Ruhland, M.K.; Kersten, K.; Abushawish, M.A., et al. Unleashing Type-2 Dendritic Cells to Drive Protective Antitumor CD4(+) T Cell Immunity. Cell 2019, 177, 556-571 e516, doi:10.1016/j.cell.2019.02.005.

11. Bastow, C.R.; Bunting, M.D.; Kara, E.E.; McKenzie, D.R.; Caon, A.; Devi, S.; Tolley, L.; Mueller, S.N.; Frazer, I.H.; Harvey, N., et al. Scavenging of soluble and immobilized CCL21 by ACKR4 regulates peripheral dendritic cell emigration. Proceedings of the National Academy of Sciences of the United States of America 2021, 118, doi:10.1073/pnas.2025763118. 
12. Bryce, S.A.; Wilson, R.A.; Tiplady, E.M.; Asquith, D.L.; Bromley, S.K.; Luster, A.D.; Graham, G.J.; Nibbs, R.J. ACKR4 on Stromal Cells Scavenges CCL19 To Enable CCR7-Dependent Trafficking of APCs from Inflamed Skin to Lymph Nodes. Journal of immunology 2016, 196, 3341-3353, doi:10.4049/jimmunol.1501542.

13. Nibbs, R.J.; Graham, G.J. Immune regulation by atypical chemokine receptors. Nature reviews. Immunology 2013, 13, 815-829, doi:10.1038/nri3544.

14. Matti, C.; Salnikov, A.; Artinger, M.; D'Agostino, G.; Kindinger, I.; Uguccioni, M.; Thelen, M.; Legler, D.F. ACKR4 Recruits GRK3 Prior to beta-Arrestins but Can Scavenge Chemokines in the Absence of beta-Arrestins. Frontiers in immunology 2020, 11, 720, doi:10.3389/fimmu.2020.00720.

15. Zhao, X.; Yuan, C.; Wangmo, D.; Subramanian, S. Tumor-Secreted Extracellular Vesicles Regulate T-Cell Costimulation and Can Be Manipulated To Induce Tumor-Specific T-Cell Responses. Gastroenterology 2021, 10.1053/j.gastro.2021.04.036, doi:10.1053/j.gastro.2021.04.036.

16. Vasaikar, S.; Huang, C.; Wang, X.; Petyuk, V.A.; Savage, S.R.; Wen, B.; Dou, Y.; Zhang, Y.; Shi, Z.; Arshad, O.A., et al. Proteogenomic Analysis of Human Colon Cancer Reveals New Therapeutic Opportunities. Cell 2019, 177, 1035-1049 e1019, doi:10.1016/j.cell.2019.03.030.

17. Sarver, A.L.; French, A.J.; Borralho, P.M.; Thayanithy, V.; Oberg, A.L.; Silverstein, K.A.; Morlan, B.W.; Riska, S.M.; Boardman, L.A.; Cunningham, J.M., et al. Human colon cancer profiles show differential microRNA expression depending on mismatch repair status and are characteristic of undifferentiated proliferative states. BMC cancer 2009, 9, 401, doi:10.1186/1471-2407-9-401.

18. Zhao, X.; Subramanian, S. Intrinsic Resistance of Solid Tumors to Immune Checkpoint Blockade Therapy. Cancer research 2017, 77, 817-822, doi:10.1158/0008-5472.CAN-16-2379.

19. Hou, T.; Liang, D.; Xu, L.; Huang, X.; Huang, Y.; Zhang, Y. Atypical chemokine receptors predict lymph node metastasis and prognosis in patients with cervical squamous cell cancer. Gynecologic oncology 2013, 130, 181-187, doi:10.1016/j.ygyno.2013.04.015.

20. Feng, L.Y.; Ou, Z.L.; Wu, F.Y.; Shen, Z.Z.; Shao, Z.M. Involvement of a novel chemokine decoy receptor CCX-CKR in breast cancer growth, metastasis and patient survival. Clinical cancer research : an official journal of the American Association for Cancer Research 2009, 15, $2962-$ 2970, doi:10.1158/1078-0432.CCR-08-2495.

21. Ju, Y.; Sun, C.; Wang, X. Loss of atypical chemokine receptor 4 facilitates $\mathrm{C}-\mathrm{C}$ motif chemokine ligand 21-mediated tumor growth and invasion in nasopharyngeal carcinoma. Experimental and therapeutic medicine 2019, 17, 613-620, doi:10.3892/etm.2018.7007.

22. Shi, J.Y.; Yang, L.X.; Wang, Z.C.; Wang, L.Y.; Zhou, J.; Wang, X.Y.; Shi, G.M.; Ding, Z.B.; Ke, A.W.; Dai, Z., et al. CC chemokine receptor-like 1 functions as a tumour suppressor by impairing CCR7related chemotaxis in hepatocellular carcinoma. The Journal of pathology 2015, 235, 546-558, doi:10.1002/path.4450.

23. Lewandowska, P.; Wierzbicki, J.; Zawadzki, M.; Agrawal, A.; Krzystek-Korpacka, M. Biphasic Expression of Atypical Chemokine Receptor (ACKR) 2 and ACKR4 in Colorectal Neoplasms in Association with Histopathological Findings. Biomolecules 2020, 11, doi:10.3390/biom11010008.

24. Lucas, B.; White, A.J.; Ulvmar, M.H.; Nibbs, R.J.; Sitnik, K.M.; Agace, W.W.; Jenkinson, W.E.; Anderson, G.; Rot, A. CCRL1/ACKR4 is expressed in key thymic microenvironments but is dispensable for $T$ lymphopoiesis at steady state in adult mice. European journal of immunology 2015, 45, 574-583, doi:10.1002/eji.201445015.

25. Eckert, N.; Werth, K.; Willenzon, S.; Tan, L.; Forster, R. B cell hyperactivation in an Ackr4deficient mouse strain is not caused by lack of ACKR4 expression. Journal of leukocyte biology 2020, 107, 1155-1166, doi:10.1002/JLB.2MA1119-300R. 
26. Werth, K.; Hub, E.; Gutjahr, J.C.; Bosjnak, B.; Zheng, X.; Bubke, A.; Russo, S.; Rot, A.; Forster, R. Expression of ACKR4 demarcates the "peri-marginal sinus," a specialized vascular compartment of the splenic red pulp. Cell reports 2021, 36, 109346, doi:10.1016/j.celrep.2021.109346.

27. Zhao, X.; Kassaye, B.; Wangmo, D.; Lou, E.; Subramanian, S. Chemotherapy but Not the Tumor Draining Lymph Nodes Determine the Immunotherapy Response in Secondary Tumors. iScience 2020, 23, 101056, doi:10.1016/j.isci.2020.101056. 


\section{FIGURE LEGENDS}

Figure 1. ACKR4 expression in human CRC tissues and cell lines.

(A) The ACKR4 mRNA expression in the TCGA CRC data set. The normal colon tissues have a higher ACKR4 expression level than the CRC tumor tissues. The MSI-H subtype tumors have a higher ACKR4 expression level than the MSI-L/MSS subtype tumors. (B) The ACKR4 mRNA expression in another independent data set published by Vasaikar et al. (C) Immunofluorescent staining of ACKR4 in human normal colon tissues $(n=17)$ and CRC tumor tissues $(n=23$ for MSI tumors and $n=45$ for MSS tumors). The representative micrographs showed the low and high ACKR4 expression cases. (D) The survival curve of CRC patients with high and low ACKR4 expression (The TCGA data set, the median value of ACKR4 expression was used as the cutoff point). (E) Western-blotting analysis of ACKR4 expression in human CRC cell lines $(n=3)$. The ACKR4 expression level was normalized to the Beta-actin expression level.

Figure 2. ACKR4 expression and tumor development.

(A) Western-blotting analysis of ACKR4 knockdown in the MC38 tumor cell line. (B) Knockdown of ACKR4 accelerated MC38 tumor growth in naïve C57BL/6J mice ( $n=5$ for the tumor growth curve and $n=10$ for the survival curve). (C) The induction and confirmation of ACKR4 knockdown in transgenetic mice. Doxycycline treatment for three weeks significantly reduced ACKR4 expression in the mouse subcutaneous connective tissue. (D) Knockdown of ACKR4 in the host mice did not significantly affect MC38 tumor growth $(n=5)$.

Figure 3. ACKR4 expression and tumor immune infiltration.

(A-B) The immune profiles of CRC cases in the TCGA data set generated by the CIBERSORT algorithm. High ACKR4 expression is associated with higher total immune cells, T cells, and DCs infiltration. (C) Immunofluorescence analysis of CD3 and CD11c on human CRC tissues. High ACKR4 expression is associated with high T-cell $\left(C D 3^{+}\right)$but not dendritic cell $\left(C D 11 c^{+}\right)$ 
infiltration ( $n=68)$. (D) FACS analysis on tumor-infiltrating T cells on MC38 tumor models. ACKR4 knockdown MC38 tumors have fewer $\mathrm{CD}^{+} \mathrm{T}$ cells in their tumor microenvironment. The percentage of exhausted CD4 ${ }^{+} \mathrm{T}$ cells is higher in the ACKR4 knockdown tumors than in the controls $(n=5-6)$.

Figure 4. ACKR4 expression and DCs migration and T-cell priming.

(A) Enriched CD45.1 $1^{+}$DCs were injected into the MC38 tumor microenvironment and analyzed in TdLNs 1 day post-injection. ACKR4 knockdown in MC38 tumor cells impaired DCs migration from the tumor microenvironment to the TdLNs $(n=4)$. (B) DCs loaded with OVA antigens were injected into the MC38 tumor microenvironment, and the OVA-specific CD8 ${ }^{+} \mathrm{T}$ cells were analyzed in the TdLNs. ACKR4 knockdown in MC38 tumor cells impaired DCs mediated T-cell priming ( $n=4-5)$. (C) P15E (a tumor-associated antigen in MC38 cells) specific CD8 ${ }^{+}$T-cell counts in TdLNs of MC38 tumors with various ACKR4 expression levels ( $n=4-5)$. (D) CCL21 quantification in MC38 tumors with different ACKR4 expression levels $(n=3-4)$.

Figure 5. Immunotherapy response on MC38 tumors with different ACKR4 expression levels.

(A) The mice were treated by anti-PD-1 on days 10,14 , and 18 . The waterfall plot showed the individual tumor volume change post-treatment. The response of the ACKR4 knockdown group to anti-PD-1 treatment was worse than that of the other groups. (B) The anti-4-1BB agonist treatment showed similar results to the anti-PD-1 treatment.

Figure 6. miR-552 downregulates ACKR4 expression in CRC tumors.

(A) The sequence match between the miR-552 and ACKR4. Dual-luciferase assay confirmed that miR-552 binds with the 3'-untranslated region of ACKR4 $(n=4)$. (B) miR-552 inhibitors enhanced ACKR4 expression in HCT116 cells $(n=3)$. (C) Expression levels of ACKR4 and miR552 are negatively correlated in human CRC tumor tissues $(n=14)$. (D) A negative correlation between ACKR4 and miR-552 in the TCGA colorectal cancer data set. 


\section{Supplementary Figure 1: ACKR4 expression and tumor immune infiltration.}

(A-B) The gating strategy for tumor-infiltrating T cells and CD103 ${ }^{+}$dendritic cells. (C) ACKR4

knockdown in MC38 tumors did not significantly change the frequencies of exhausted and activated $\mathrm{CD}^{+} \mathrm{T}$ cells and $\mathrm{CD} 103^{+} \mathrm{DCs}$ in the tumor microenvironment. The ACKR4 expression on tumor cells did not alter the CD80 and CD86 expression on the CD103 ${ }^{+}$DCs ( $\left.n=5-6\right)$. (D) The ACKR4 expression on tumor cells did not change the functional status of the general T cells and DCs in the TdLNs $(n=4-5)$. 


\section{KEY RESOURCES TABLE}

\begin{tabular}{|c|c|c|}
\hline REAGENT FOR IMMUNOASSAY & SOURCE & IDENTIFIER \\
\hline Anti-human/mouse CD3 & Abcam & Cat \#: ab11089 \\
\hline Anti-human CD11c & Abcam & Cat \#: ab52632 \\
\hline Anti-human CD11c & Abcam & Cat \#: ab212508 \\
\hline Anti-human ACKR4 & Novus & Cat \#: NB100-705 \\
\hline Anti-human/mouse ACKR4 & Abcam & Cat \#: ab32564 \\
\hline Anti-human/mouse Beta-actin & Novus & Cat \#: NB600-501 \\
\hline Goat anti-Rat Secondary Antibody, Alexa Fluor 568 & Invitrogen & Cat \#: A11077 \\
\hline Goat anti-Rabbit Secondary Antibody, Alexa Fluor 488 & Invitrogen & Cat \#: A11008 \\
\hline Goat anti-Mouse Secondary Antibody, Alexa Fluor 488 & Invitrogen & Cat \#: A11001 \\
\hline Donkey anti-Goat Secondary Antibody, Alexa Fluor 488 & Invitrogen & Cat \#: A11055 \\
\hline Anti-human HLA-DR-PE (L243) & BioLegend & Cat \#: 307606 \\
\hline Anti-human CD14-Pacific Blue (63D3) & BioLegend & Cat \#: 367122 \\
\hline Anti-human CD1a-FITC (HI149) & BioLegend & Cat \#: 300104 \\
\hline Anti-human CD80-BV 510 (2D10) & BioLegend & Cat \#: 305234 \\
\hline Anti-human CD45-APC/Cy7 (HI30) & BioLegend & Cat \#: 304014 \\
\hline Anti-human CD11c-APC (3.9) & BioLegend & Cat \#: 301614 \\
\hline Anti-mouse CD4-BV510 (GK1.5) & BioLegend & Cat \#: 100449 \\
\hline Anti-mouse/human CD11b-FITC (M1/70) & BioLegend & Cat \#: 101205 \\
\hline Anti-mouse NK-1.1-FITC (PK136) & BioLegend & Cat \#: 108705 \\
\hline Anti-mouse CD19-FITC (6D5) & BioLegend & Cat \#: 115505 \\
\hline Anti-mouse PD-1-PerCP/Cy5.5 (29F.1A12) & BioLegend & Cat \#: 135208 \\
\hline Anti-mouse CD3-PE/Cy7 (17A2) & BioLegend & Cat \#: 100220 \\
\hline Anti-mouse CD45-APC/Cy7 (30-F11) & BioLegend & Cat \#: 103115 \\
\hline Anti-mouse CD366 (Tim-3)-BV421 (RMT3-23) & BioLegend & Cat \#: 119723 \\
\hline Anti-mouse CD4-BV510 (GK1.5) & BioLegend & Cat \#: 100449 \\
\hline Anti-mouse CD3-FITC (17A2) & BioLegend & Cat \#: 100204 \\
\hline Anti-mouse CD86-PE (GL-1) & BioLegend & Cat \#: 105007 \\
\hline Anti-mouse CD11c-PerCP/Cy5.5 (N418) & BioLegend & Cat \#: 117328 \\
\hline Anti-mouse CD80-PE/Cy7 (16-10A1) & BioLegend & Cat \#: 104734 \\
\hline Anti-mouse/human CD11b-APC (M1/70) & BioLegend & Cat \#: 101212 \\
\hline Anti-mouse I-A/I-E-APC/Cy7 (M5/114.15.2) & BioLegend & Cat \#: 107628 \\
\hline Anti-mouse CD45.1-BV421 (A20) & BioLegend & Cat \#: 110732 \\
\hline Anti-CD8 (Mouse) mAb-PE (Monoclonal Antibody) & $\begin{array}{l}\mathrm{MBL} \\
\text { International }\end{array}$ & Cat \#: D271-5 \\
\hline Anti-mouse CD62L-PE/Cy7 (MEL-14) & BioLegend & Cat \#: 104418 \\
\hline Anti-mouse CD8a-APC/Cy7 (53-6.7) & BioLegend & Cat \#: 100714 \\
\hline Anti-mouse CD45-Pacific Blue (30-F11) & BioLegend & Cat \#: 103126 \\
\hline Anti-mouse CD103-Pacific Blue (2E7) & BioLegend & Cat \#: 121418 \\
\hline Anti-mouse/human CD11b-APC (M1/70) & BioLegend & Cat \#: 101212 \\
\hline Anti-mouse CD45-BV510 (30-F11) & BioLegend & Cat \#: 103138 \\
\hline Anti-human/mouse Granzyme B-AF647 (GB11) & BioLegend & Cat \#: 515406 \\
\hline Anti-mouse IFN- $\gamma$ - PerCP/Cy5.5 (XMG1.2) & BioLegend & Cat \#: 505822 \\
\hline Anti-mouse CD3-PE (17A2) & BioLegend & Cat \#: 100206 \\
\hline Anti-mouse CD45-PE (30-F11) & BioLegend & Cat \#: 103106 \\
\hline Anti-mouse CD3-APC (17A2) & BioLegend & Cat \#: 100236 \\
\hline Tetramer/BV421 - H-2 Kb OVA (SIINFEKL) & MBL & Cat \#: TB-5001-4 \\
\hline
\end{tabular}




\begin{tabular}{|c|c|c|}
\hline & International & \\
\hline T-Select I-Ab OVA 323-339 Tetramer-APC & $\begin{array}{l}\text { MBL } \\
\text { International }\end{array}$ & Cat \#: TS-M710-2 \\
\hline H-2Kb MuLV p15E Tetramer-KSPWFTTL-APC & $\begin{array}{l}\text { MBL } \\
\text { International }\end{array}$ & Cat \#: TB-M507-2 \\
\hline Rat IgG1, k Isotype Ctrl-Pacific Blue (RTK2071) & BioLegend & Cat \#: 400419 \\
\hline Rat IgG1, k Isotype Ctrl-PE/Cy7 (RTK2071) & BioLegend & Cat \#: 400415 \\
\hline Mouse IgG1, k Isotype Ctrl-PE/Cy7 (MOPC-21) & BioLegend & Cat \#: 400125 \\
\hline Mouse IgG1, k Isotype Ctrl-PE (MOPC-21) & BioLegend & Cat \#: 400111 \\
\hline Mouse IgG1, k Isotype Ctrl-BV510 (MOPC-21) & BioLegend & Cat \#: 400171 \\
\hline Mouse IgG1, k Isotype Ctrl-PerCP/Cy5.5 (MOPC-21) & BioLegend & Cat \#: 400149 \\
\hline Rat IgG2a, k Isotype Ctrl-PerCP/Cy5.5 (RTK2758) & BioLegend & Cat \#: 400531 \\
\hline Zombie Green TM Fixable Viability Kit & BioLegend & Cat \#: 423111 \\
\hline Cyto-Fast $^{\mathrm{TM}}$ Fix/Perm Buffer Set & BioLegend & Cat \#: 426803 \\
\hline True-Nuclear ${ }^{\mathrm{TM}}$ Transcription Factor Buffer Set & BioLegend & Cat \#: 424401 \\
\hline CCL21 Mouse ELISA Kit & Abcam & Cat \#: ab208985 \\
\hline TREATMENT & SOURCE & IDENTIFIER \\
\hline InVivoPlus anti-mouse PD-1 & Bio X Cell & Cat \#: BP0146 \\
\hline InVivoMAb anti-mouse 4-1BB (CD137) & Bio X Cell & Cat \#: BE0239 \\
\hline InVivoPlus rat IgG2a isotype control & Bio X Cell & Cat \#: BP0089 \\
\hline REAGENT FOR DNA/RNA EXPERIMENTS & SOURCE & IDENTIFIER \\
\hline psiCHECK ${ }^{\mathrm{TM}}-2$ Vectors & Promega & Cat \#: C8021 \\
\hline pPACKH1-XL packaging mix & SBI & Cat \#: LV510A-1 \\
\hline Lipofectamine $^{\text {TM }} 3000$ Transfection Reagent & Invitrogen & Cat \#: L3000001 \\
\hline mirVana ${ }^{\mathrm{TM}}$ miRNA Isolation Kit & Invitrogen & Cat \#: AM1560 \\
\hline miScript II RT Kit & Qiagen & Cat \#: 218161 \\
\hline LightCycler® 480 SYBR Green I Master & Roche & $\begin{array}{l}\text { Cat \#: } \\
04707516001\end{array}$ \\
\hline OTHER REAGENTS & SOURCE & IDENTIFIER \\
\hline Advanced DMEM F/12 & $\begin{array}{l}\text { Thermo } \\
\text { Fisher } \\
\text { Scientific }\end{array}$ & Cat \#: 12634-010 \\
\hline Glutamax & $\begin{array}{l}\text { Life } \\
\text { Technologie } \\
\mathrm{s}\end{array}$ & Cat \#: 35050-061 \\
\hline N-Acetylcysteine & $\begin{array}{l}\text { Sigma } \\
\text { Aldrich }\end{array}$ & Cat \#: A9165-SG \\
\hline Growth factor reduced Matrigel & $\mathrm{BD}$ & Cat \#: 354230 \\
\hline Y-27632 (Rho Kinase inhibitor) & $\begin{array}{l}\text { EMD } \\
\text { Millipore }\end{array}$ & Cat \#: 688001 \\
\hline EGF & Invitrogen & Cat \#: PMG8043 \\
\hline L-WRN cells & ATCC & Cat \#: CRL-3276 \\
\hline SB202190 & $\begin{array}{l}\text { Sigma } \\
\text { Aldrich }\end{array}$ & Cat \#: S7067 \\
\hline B27 supplement & Invitrogen & Cat \#: 17504-044 \\
\hline N2 Supplement & Invitrogen & Cat \#: 17502-048 \\
\hline TrypLE Express & Invitrogen & Cat \#: 12605-036 \\
\hline Primocin & Invivogen & Cat \#: ant-pm-1 \\
\hline
\end{tabular}




\begin{tabular}{|c|c|c|}
\hline Gastrin & $\begin{array}{l}\text { Sigma } \\
\text { Aldrich }\end{array}$ & Cat \#: G9145 \\
\hline Nicotinamide & $\begin{array}{l}\text { Sigma } \\
\text { Aldrich }\end{array}$ & Cat \#: N0636 \\
\hline A-83-01 & Tocris & Cat \#: 2939 \\
\hline Prostaglandin E2 & $\begin{array}{l}\text { Cayman } \\
\text { Chemicals }\end{array}$ & Cat \#: 14010-1 \\
\hline Matrigel $^{\mathrm{TM}}$ Membrane Matrix & Corning & Cat \#: 354234 \\
\hline H\&E Staining Kit & Abcam & Cat \#: ab245880 \\
\hline ProLong ${ }^{\mathrm{TM}}$ Gold Antifade Mountant & Invitrogen & Cat \#: P36934 \\
\hline AR6 Buffer & PerkinElmer & $\begin{array}{l}\text { Cat \#: } \\
\text { AR600250ML }\end{array}$ \\
\hline Dynabeads $^{\mathrm{TM}}$ Mouse DC (Dendritic Cell) Enrichment & Invitrogen & Cat \#: 11429D \\
\hline CellXVivo Mouse Dendritic Cell Differentiation Kit & $\begin{array}{l}\text { R\&D } \\
\text { Systems }\end{array}$ & Cat \#: CDK008 \\
\hline MICE & SOURCE & IDENTIFIER \\
\hline ACKR4-shRNA mice & Mirimus Inc & NA \\
\hline C57BL/6 mice & $\begin{array}{l}\text { The Charles } \\
\text { River } \\
\text { Laboratories }\end{array}$ & Stock No: 027 \\
\hline SOFTWARE & SOURCE & IDENTIFIER \\
\hline GraphPad Prism 6 & $\begin{array}{l}\text { GraphPad } \\
\text { Software }\end{array}$ & $\begin{array}{l}\text { https://www.graphp } \\
\text { ad.com/ }\end{array}$ \\
\hline FlowJo & $\begin{array}{l}\text { BD } \\
\text { Biosciences }\end{array}$ & $\begin{array}{l}\text { https://www.flowjo.c } \\
\text { om/ }\end{array}$ \\
\hline CIBERSORT & NA & $\begin{array}{l}\text { https://cibersort.sta } \\
\text { nford.edu/ }\end{array}$ \\
\hline
\end{tabular}


A

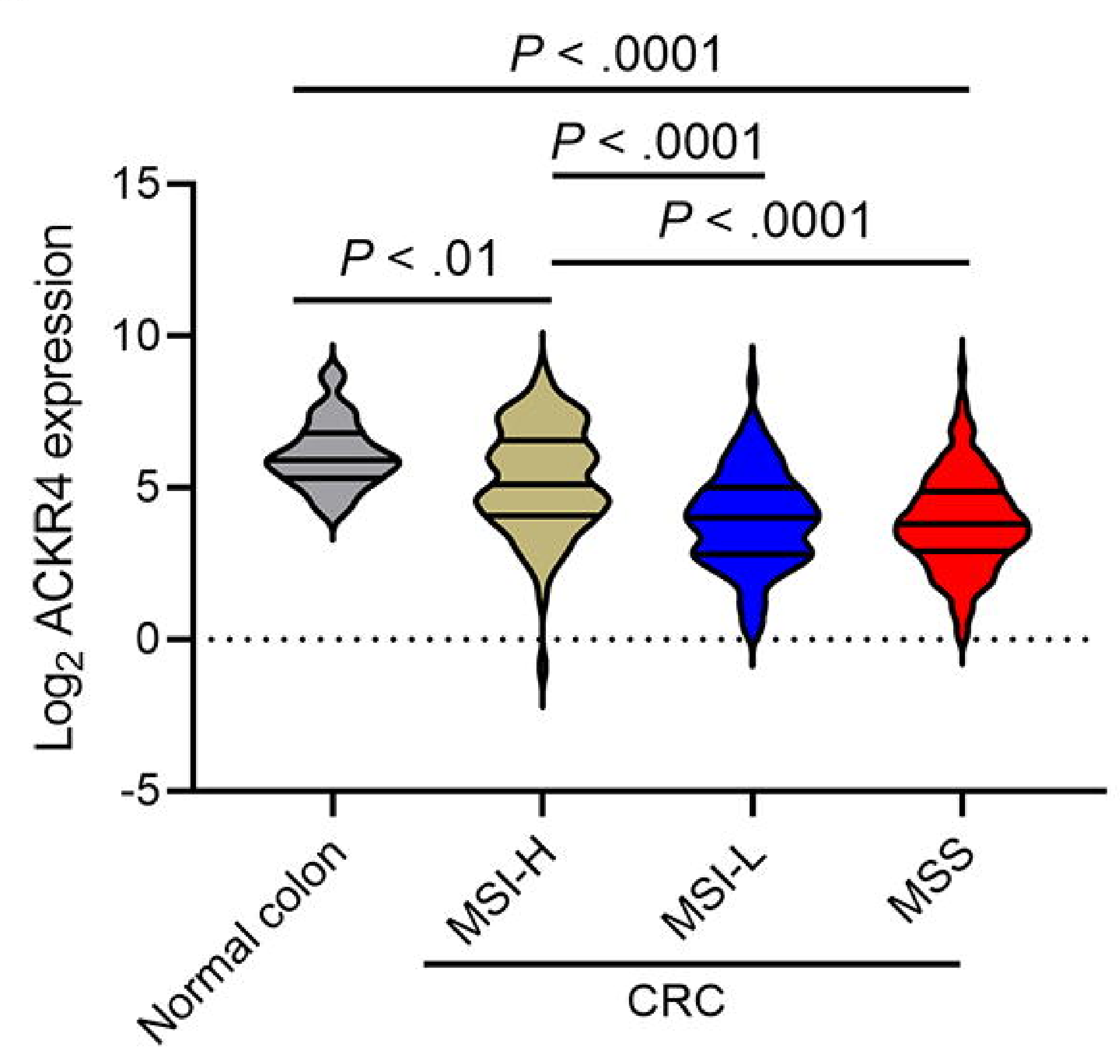

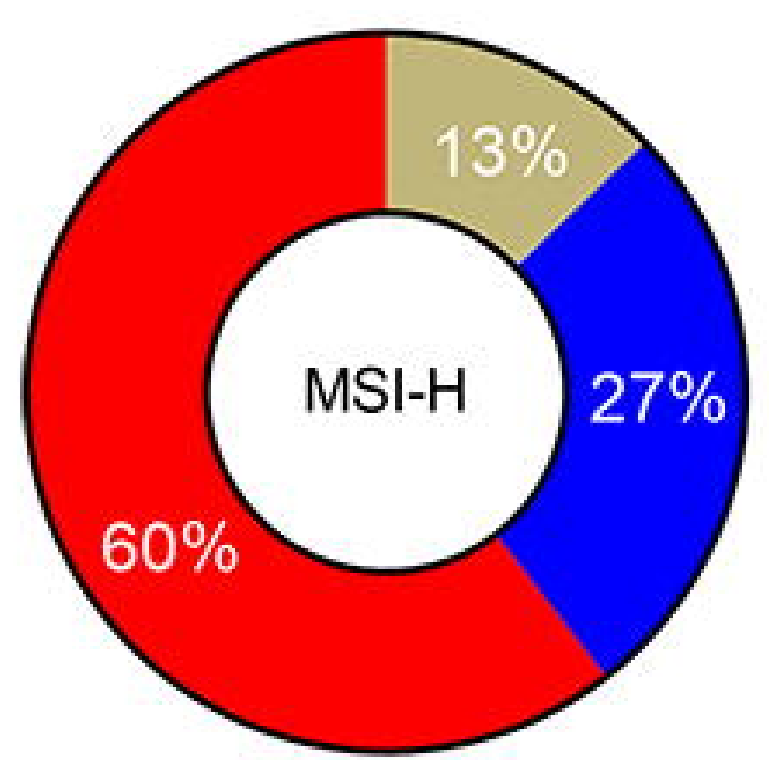

ACKR4 expression

므 Lower $1 / 3$

ㅁ. Medium $1 / 3$

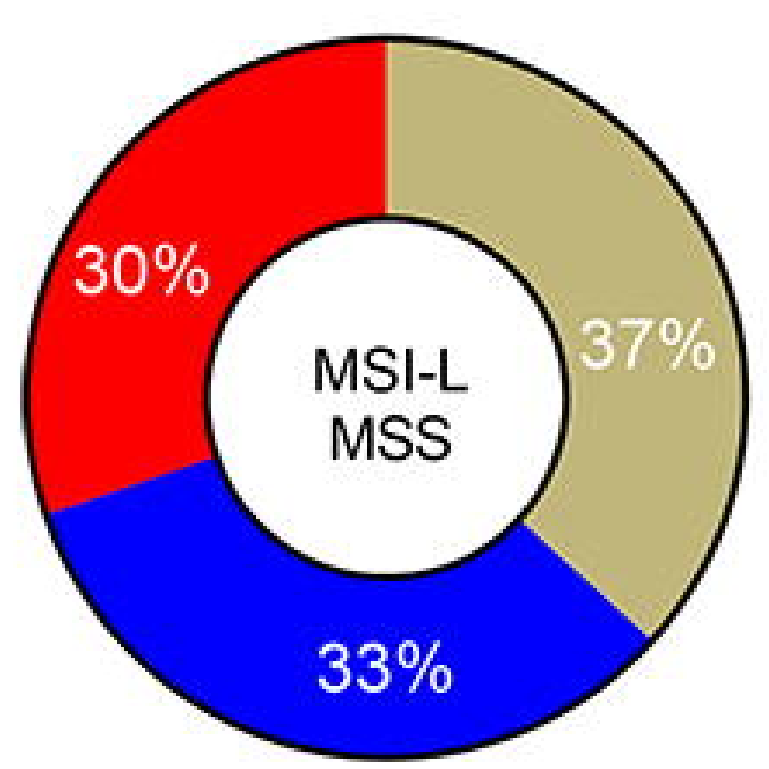

Chi-square test $P<0001$ 口 Lower $1 / 3$ Medium $1 / 3$
B

Vasaikar et al.

Cell 2019 dataset

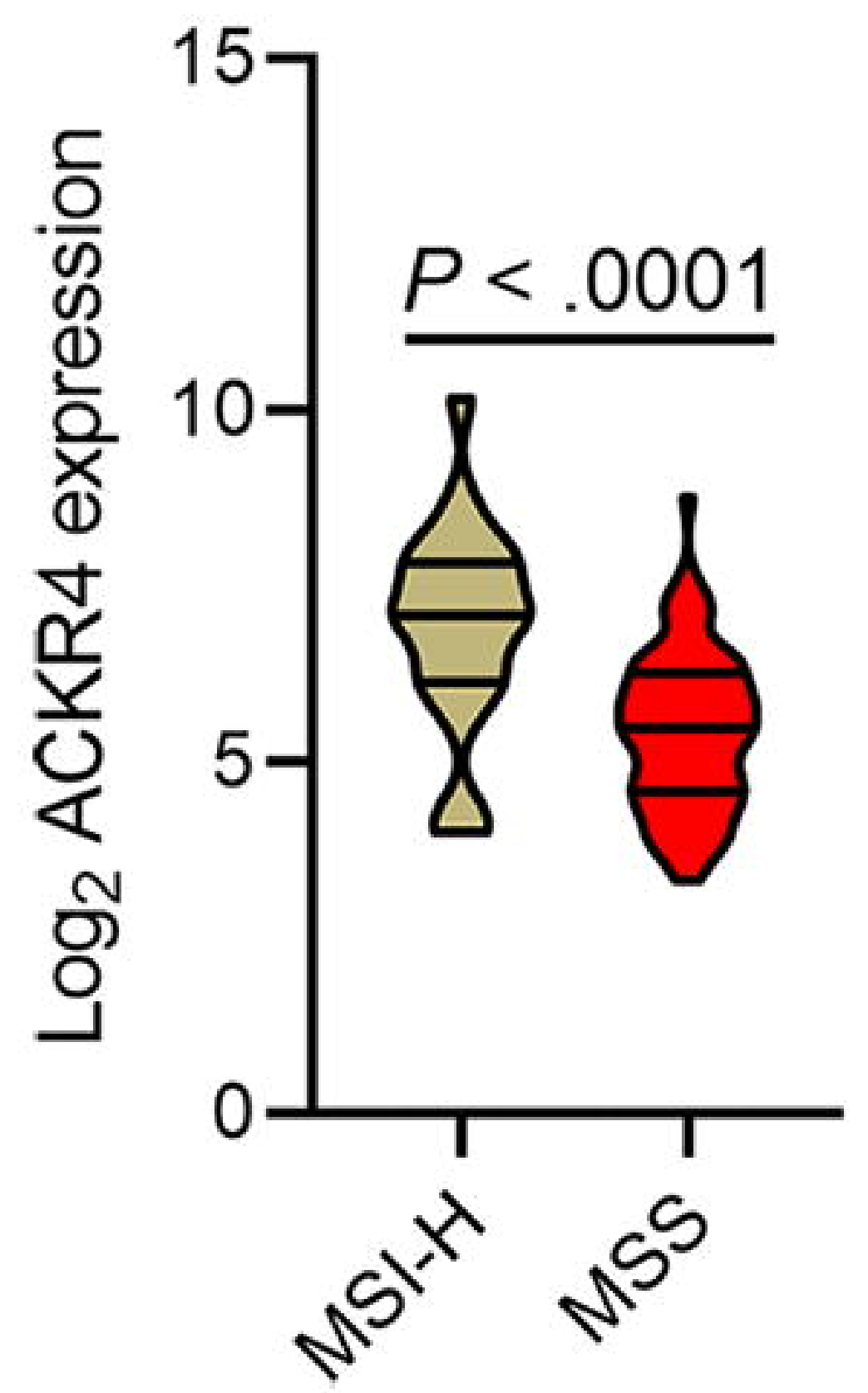

Vasaikar et al. Cell 2019 dataset

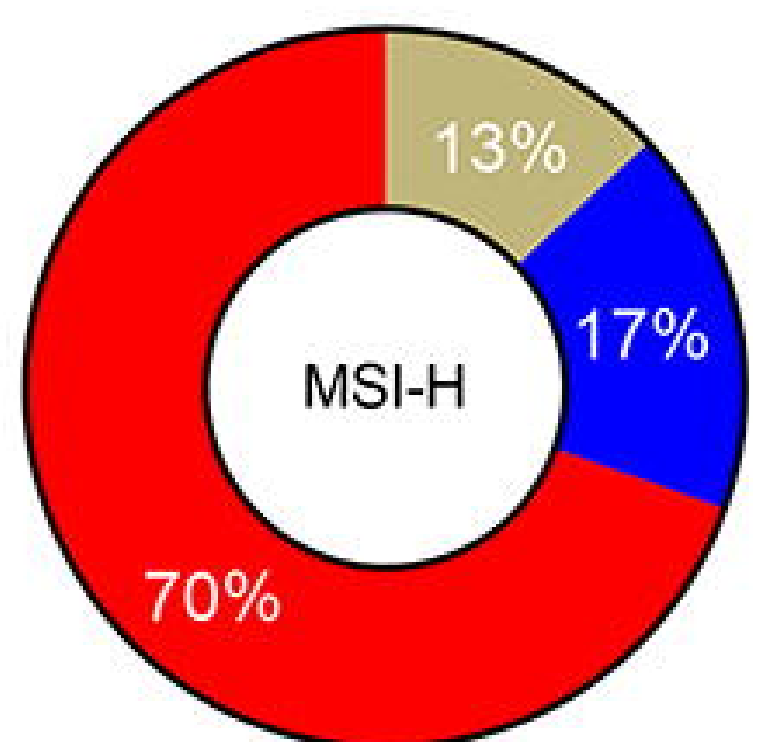

KR4 expression 口 Lower $1 / 3$ 口 Upper

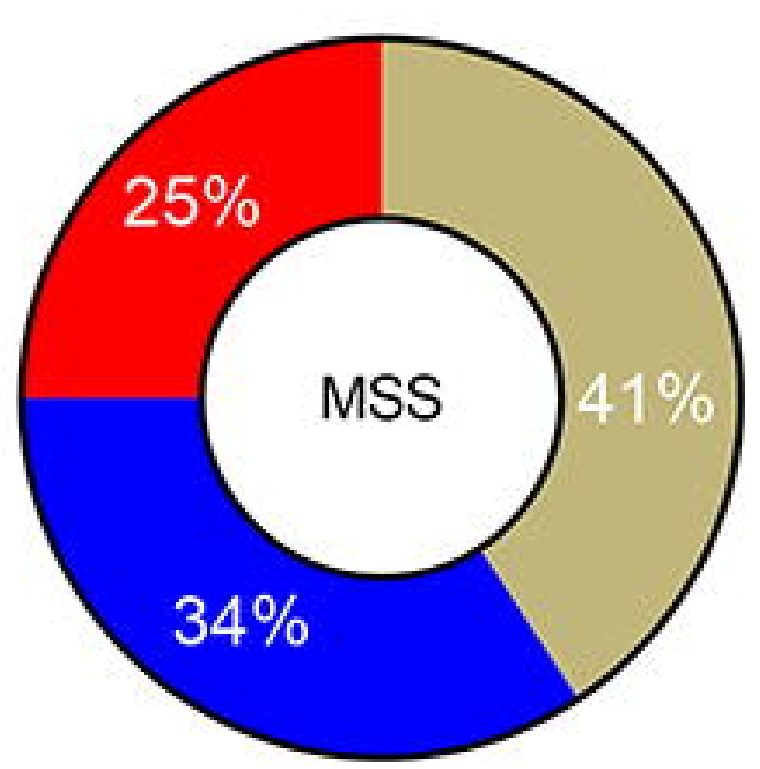

ACKR4 expression $\square$ Lower $1 / 3$ U Upper $1 / 3$

Chi-square test $P<.001$
C

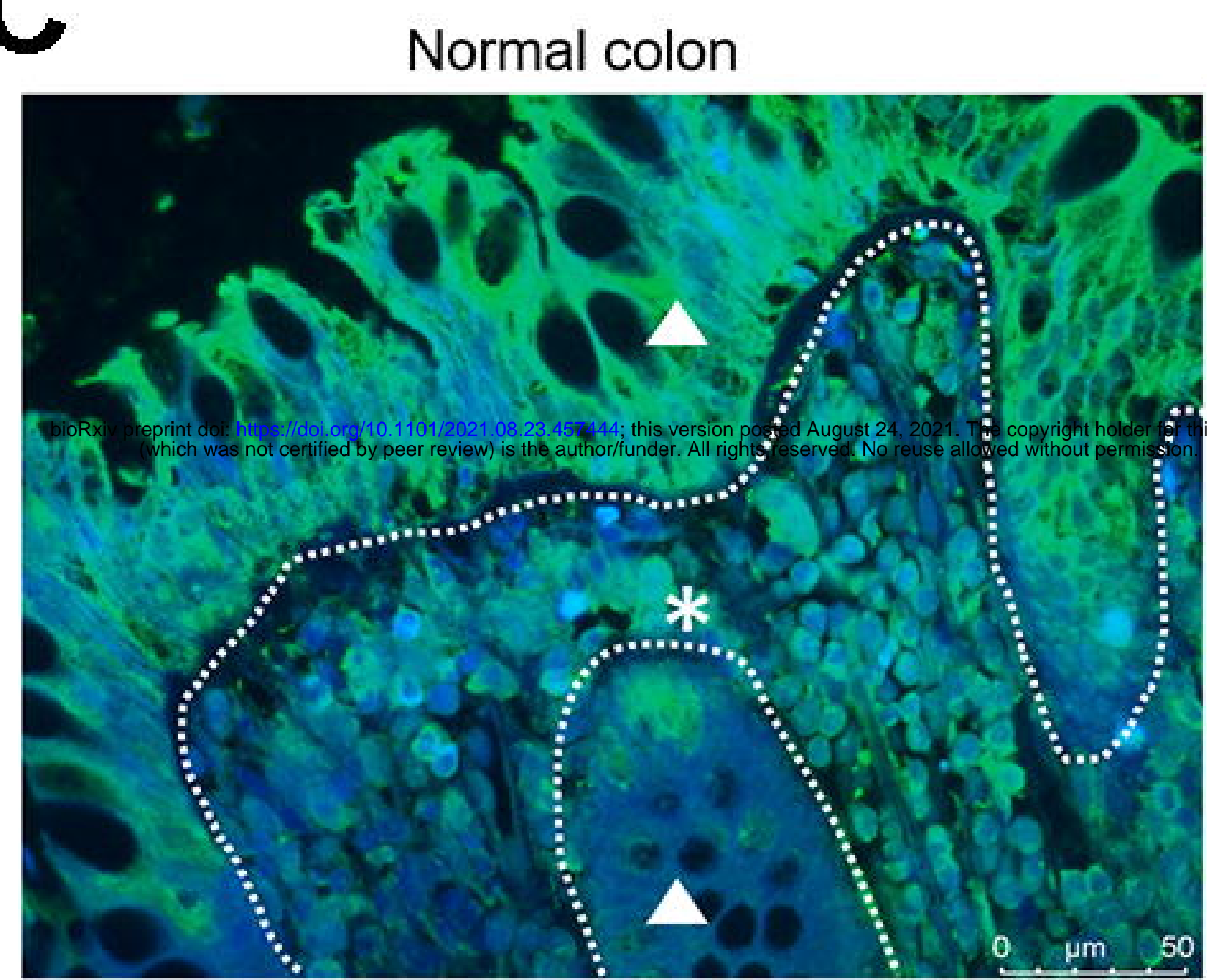

DAPI ACKR4

D

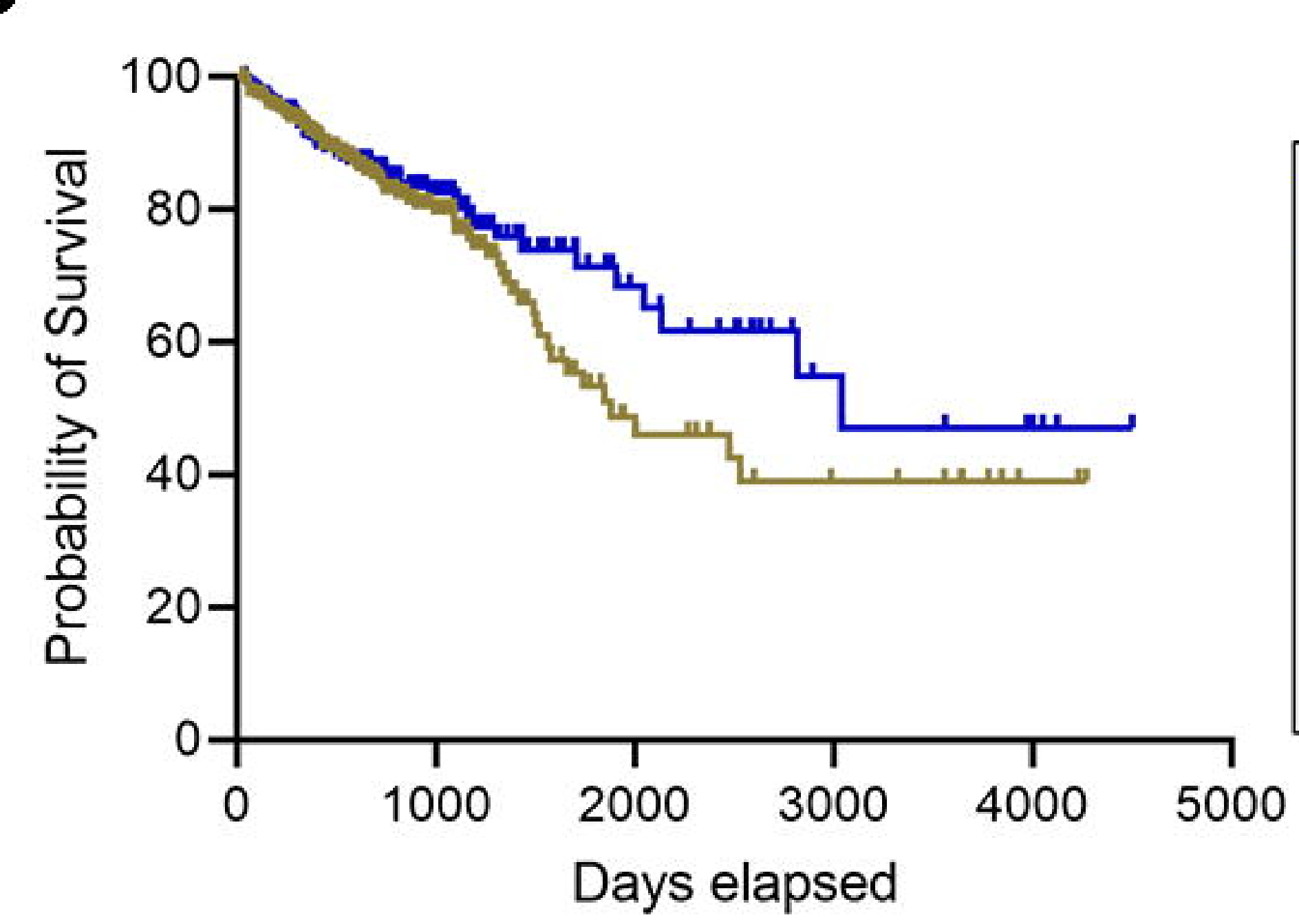

Colon cancer with high ACKR4 expression

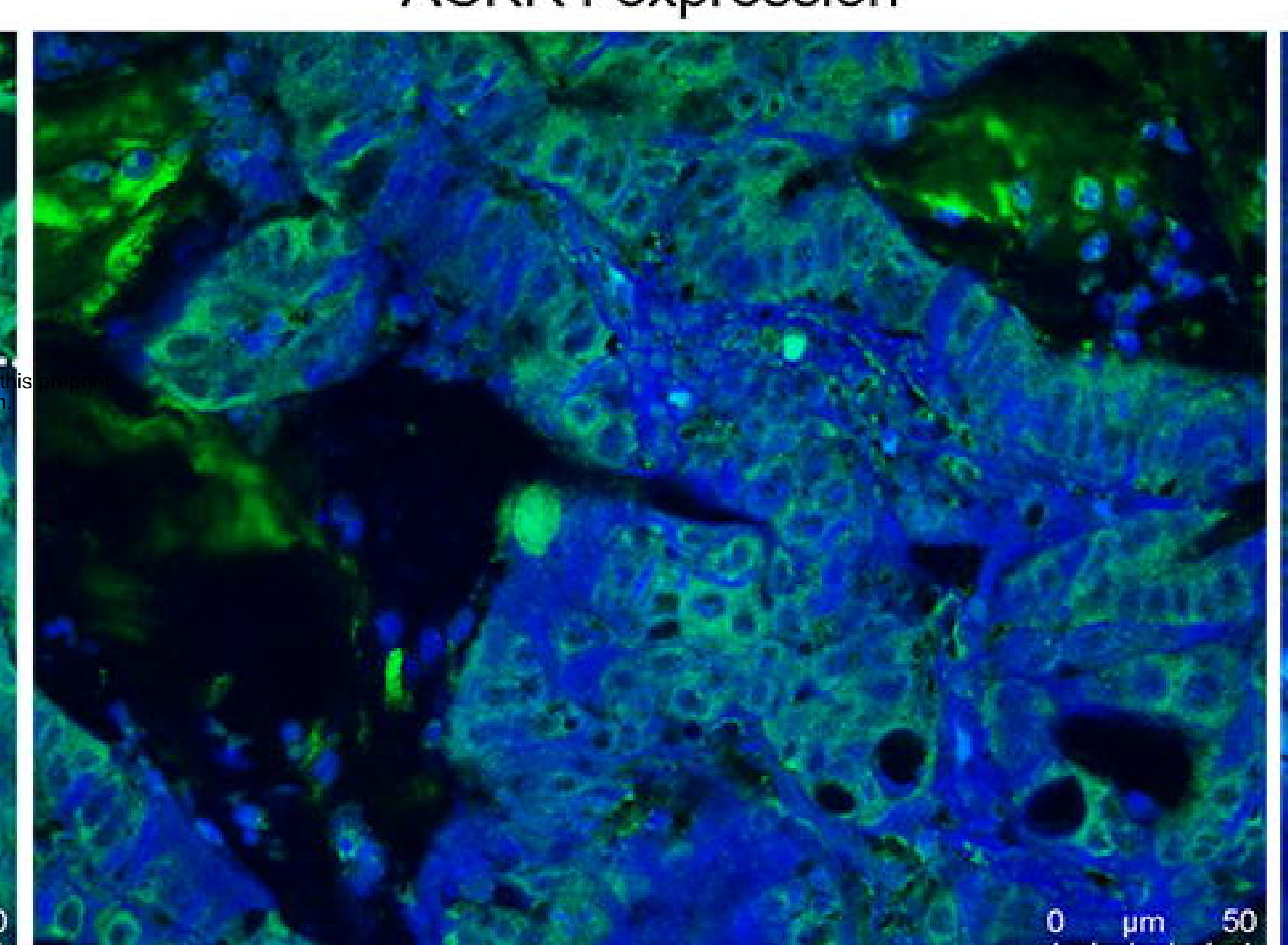

ACKR4-upper 1/2

ACKR4-lower 1/2

Log-rank test

$P=0.161$

Median survival time

ACKR4-upper 1/2: 3042 days

ACKR4-lower 1/2: 1881 days
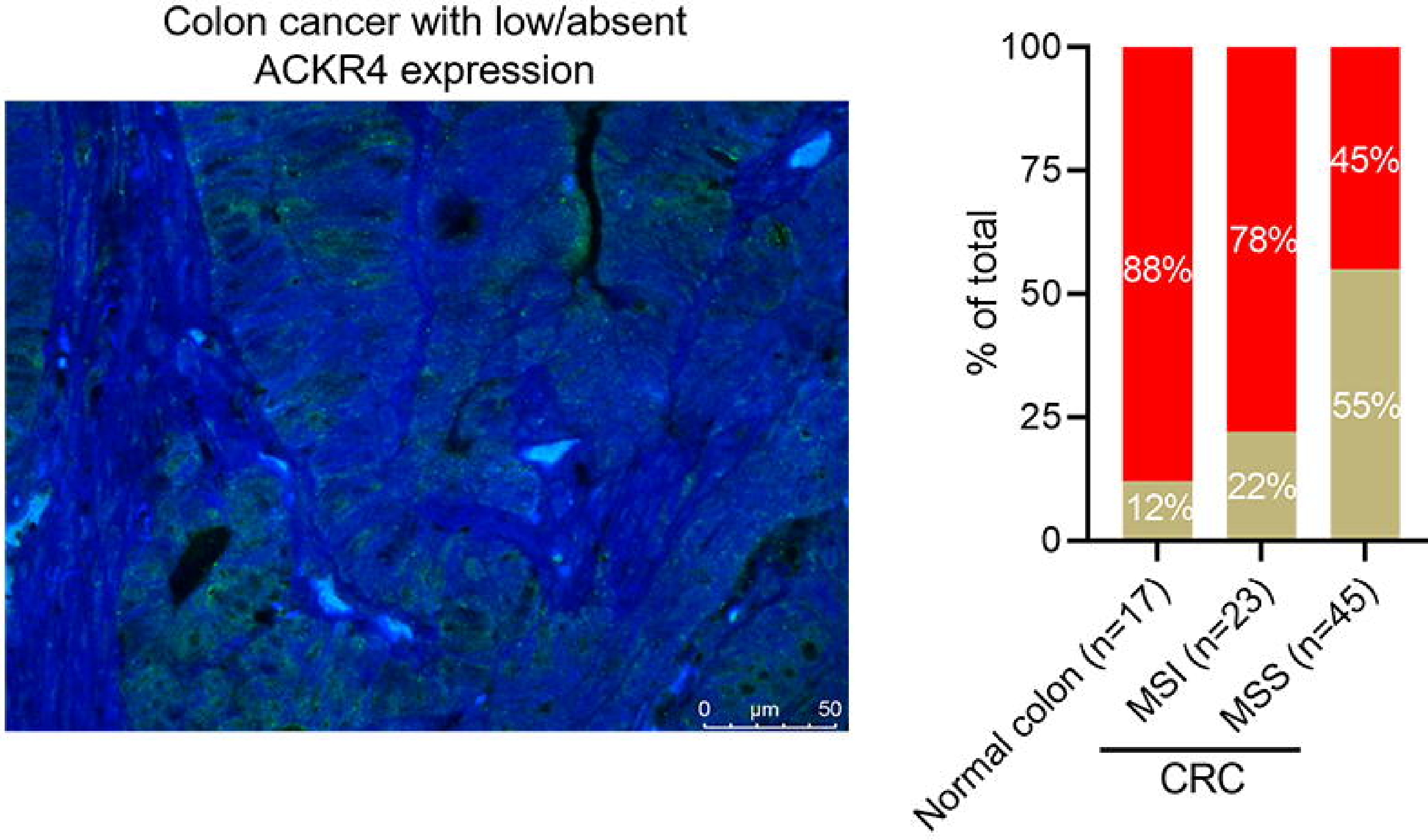

Chi-square test $P<.0001$

- ACKR4-High

aCKR4-Low/Absent
E

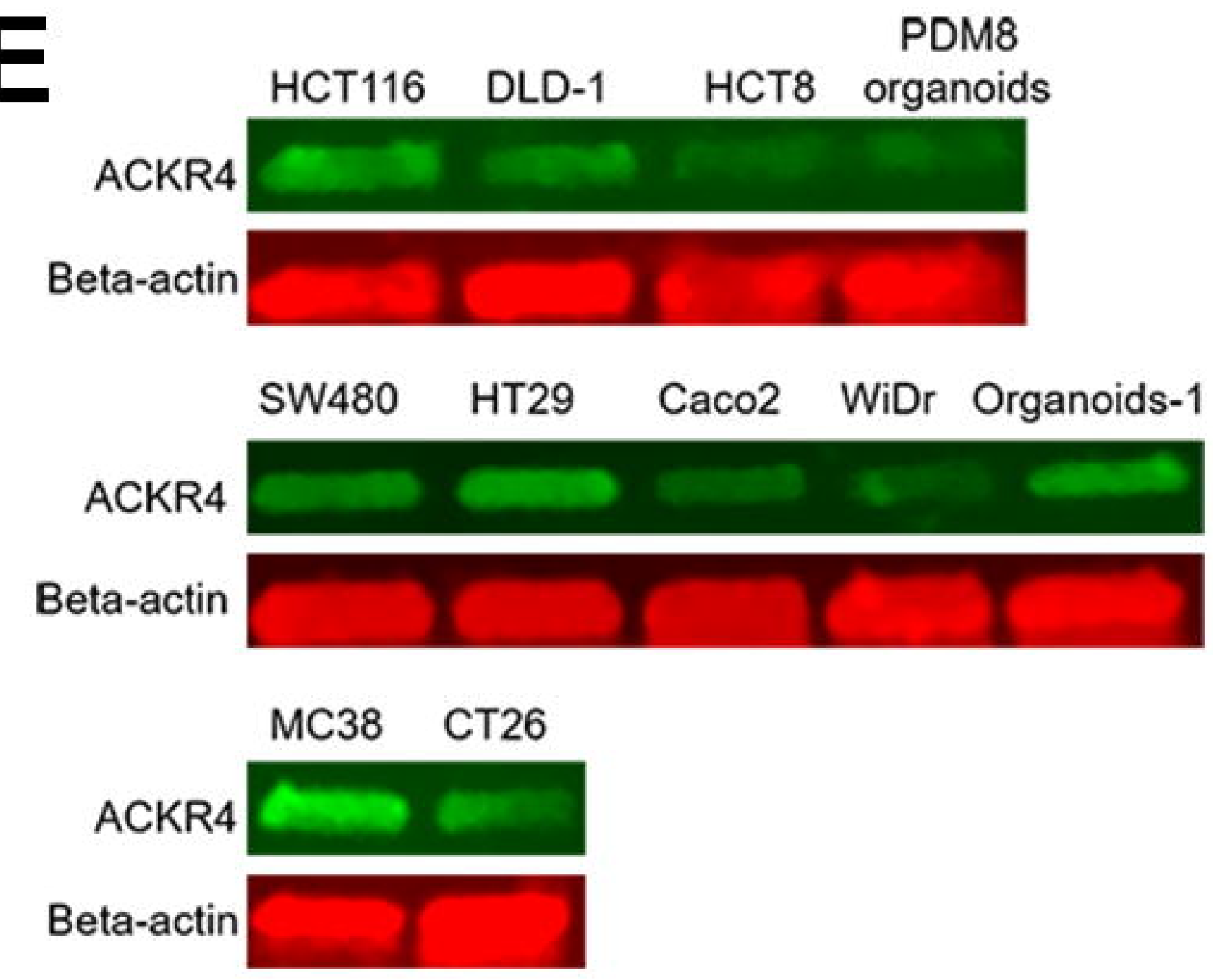

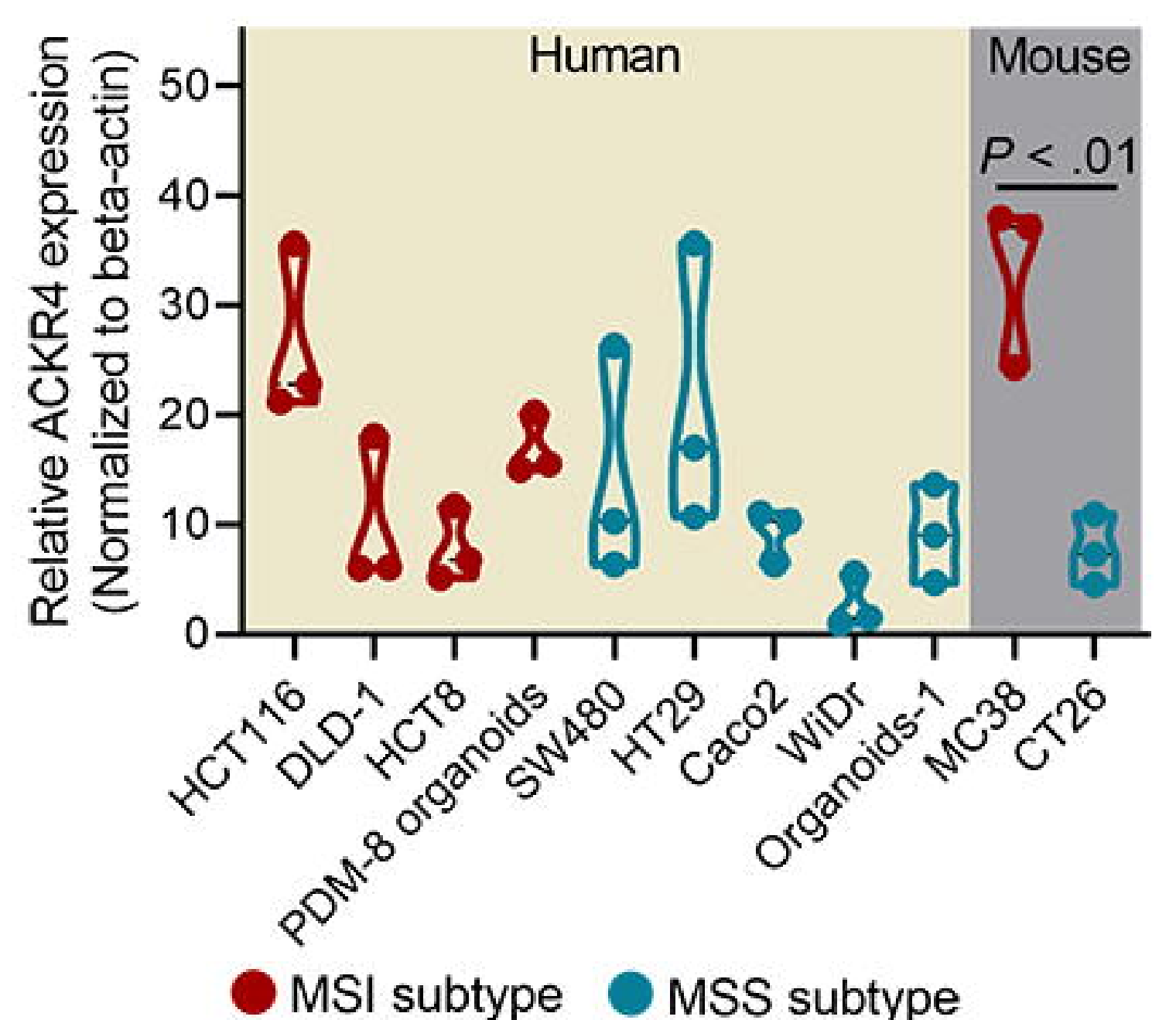




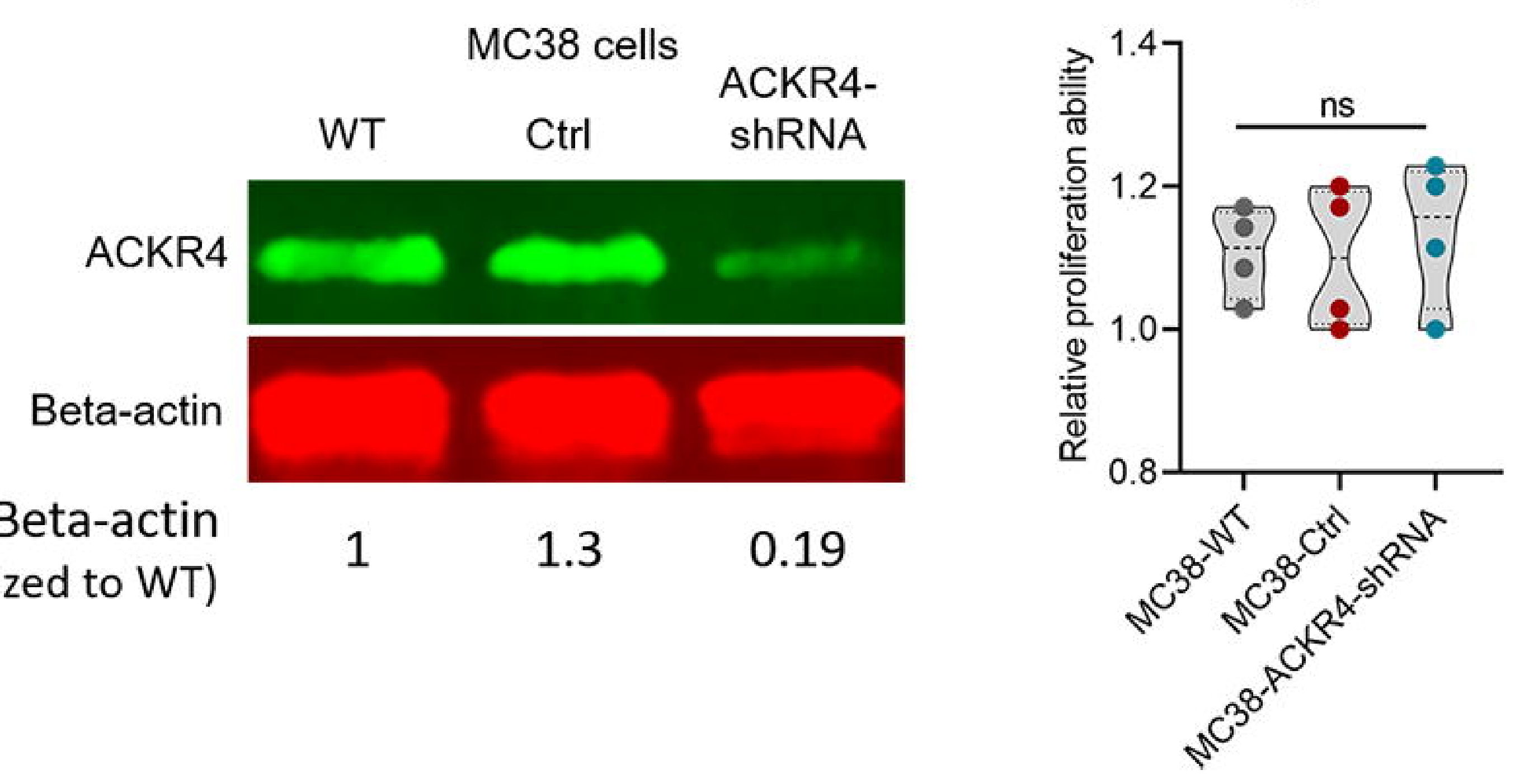

$B$ мс38-Wт

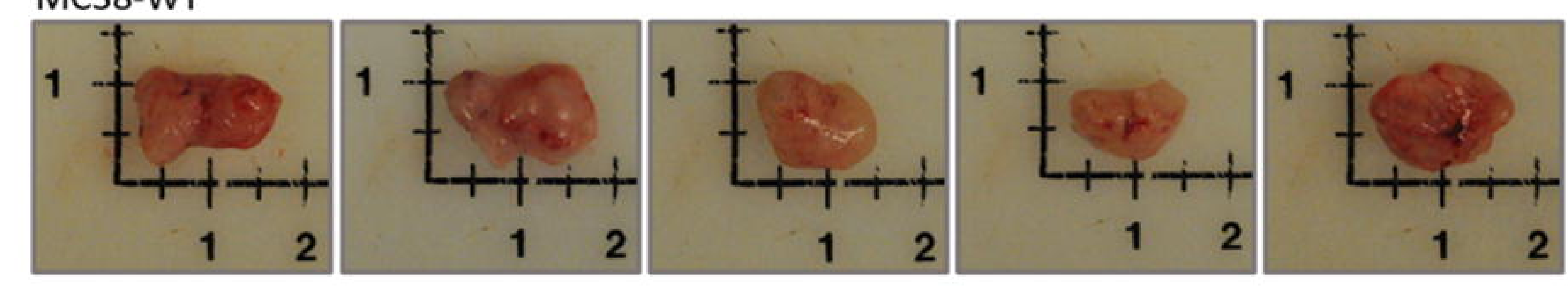

MC38-Ctrl

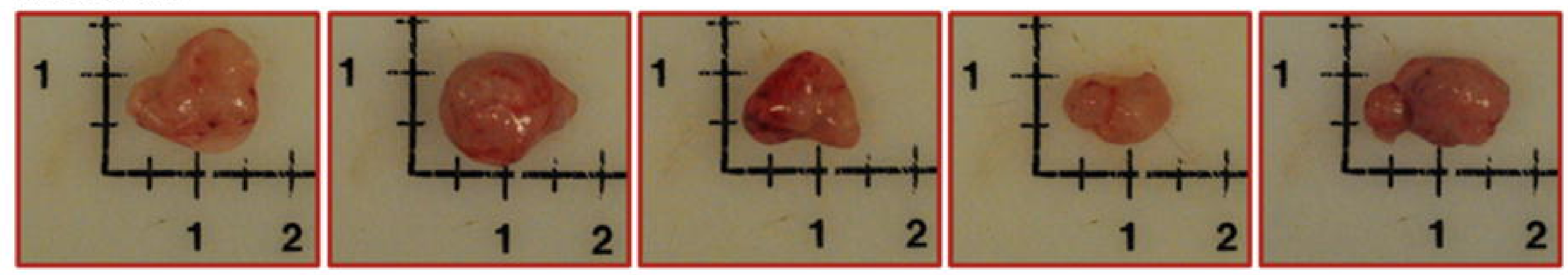

MC38-ACKR4-shRNA
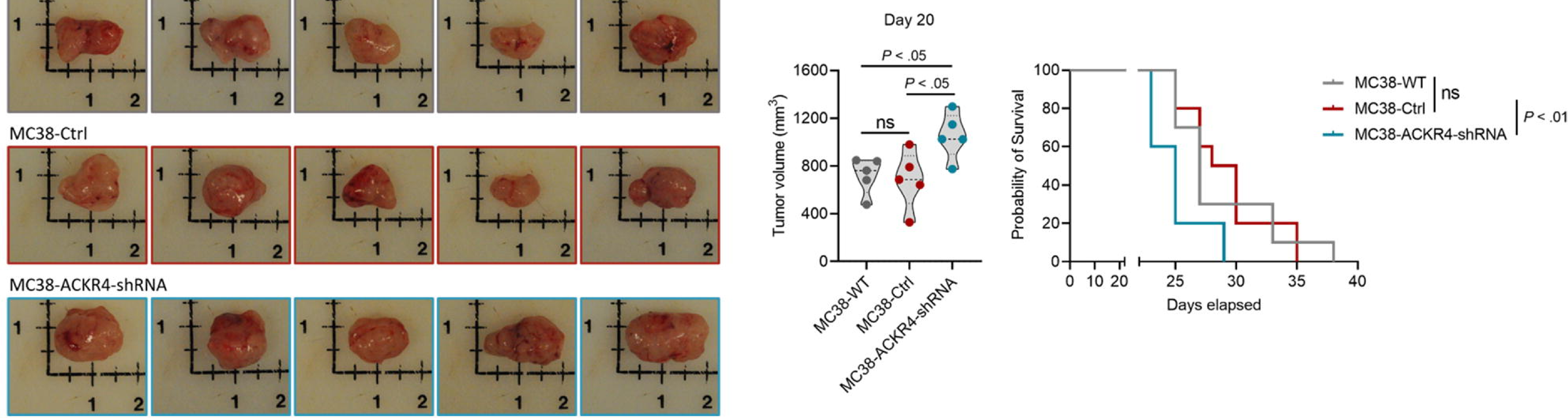

C

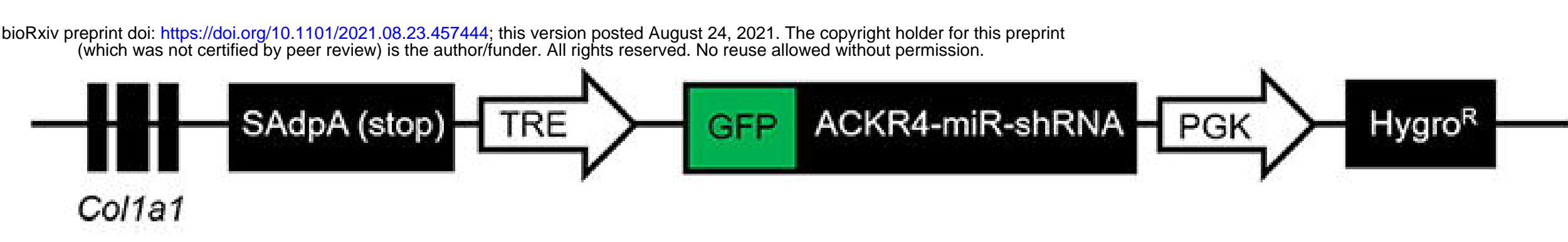

ACKR4

Beta-actin

D

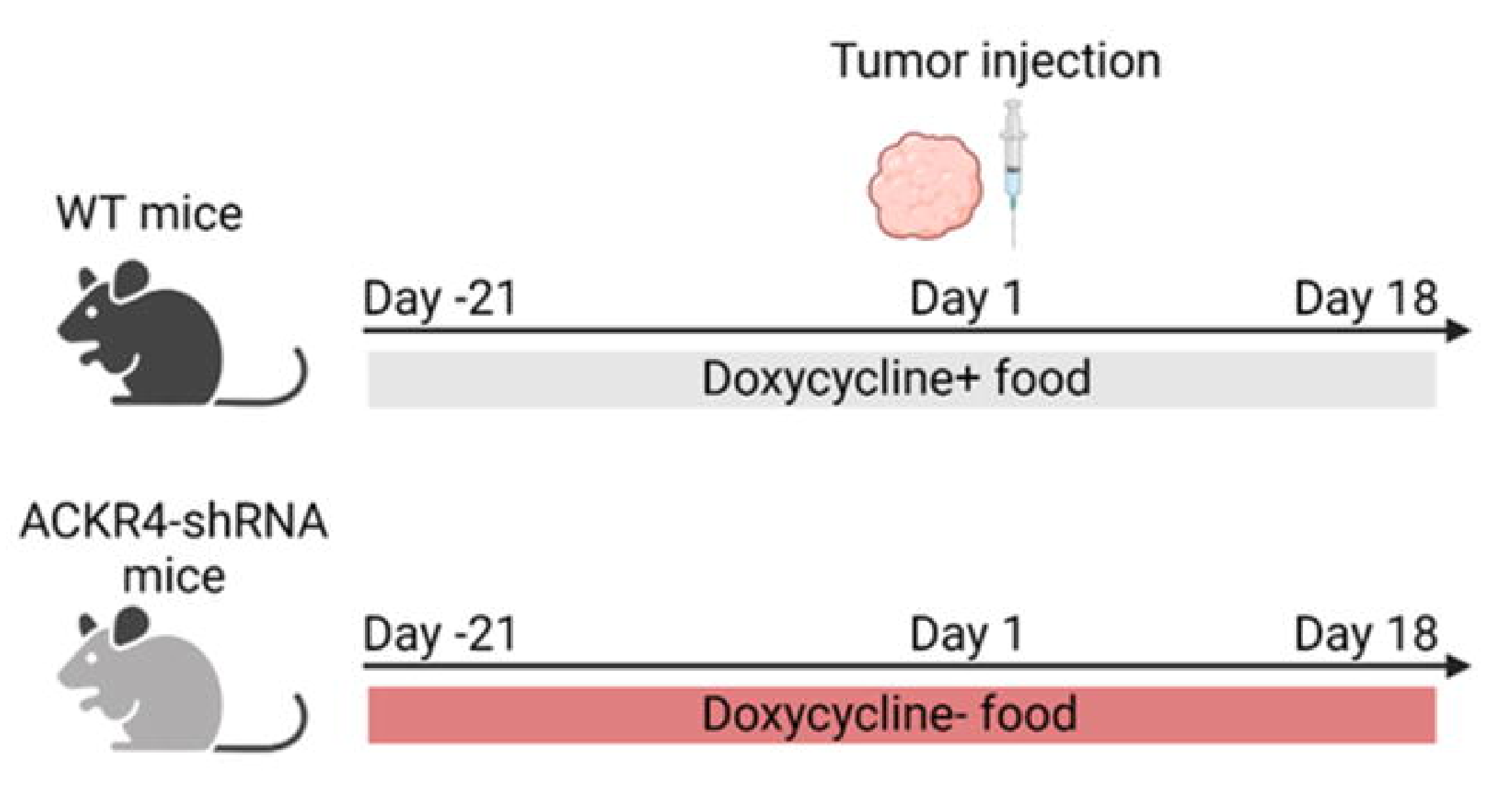

ACKR4-shRNA

1

Day - 21

Day 1

$\stackrel{\text { Day } 18}{\longrightarrow}$
WT mice, Doxycycline+

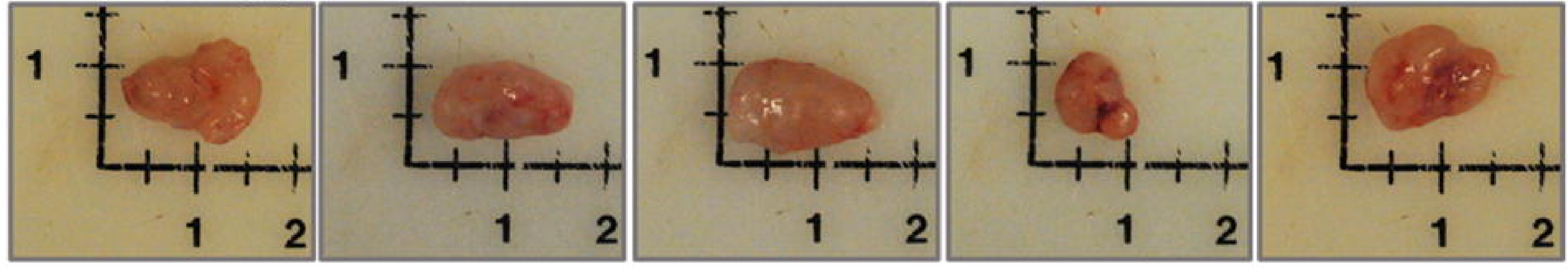

ACKR4-shRNA mice, Doxycycline-

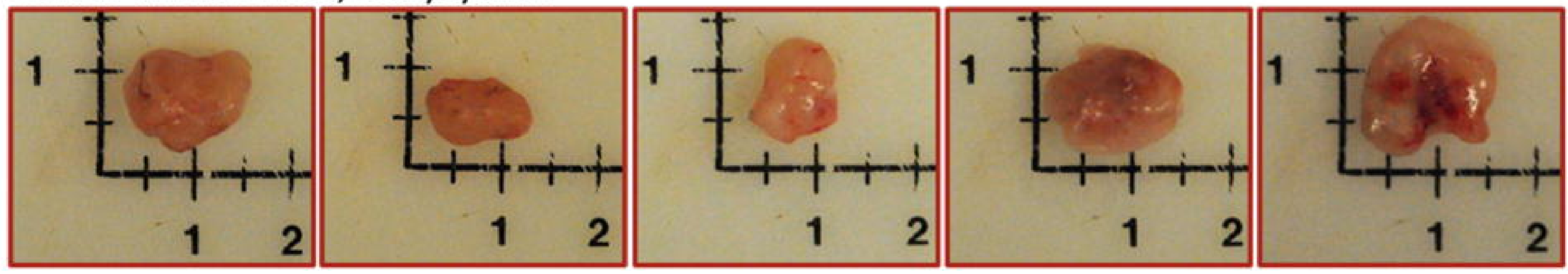

ACKR4-shRNA mice, Doxycycline+

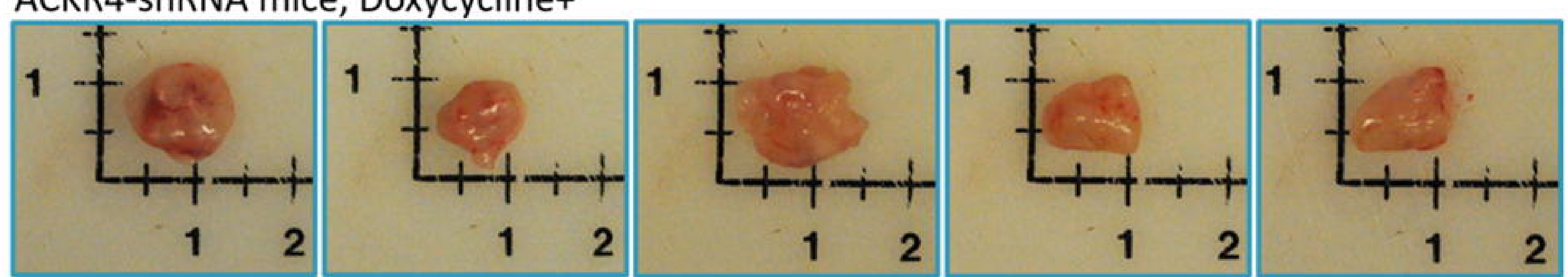

ACKR4shRNA mice Day 18
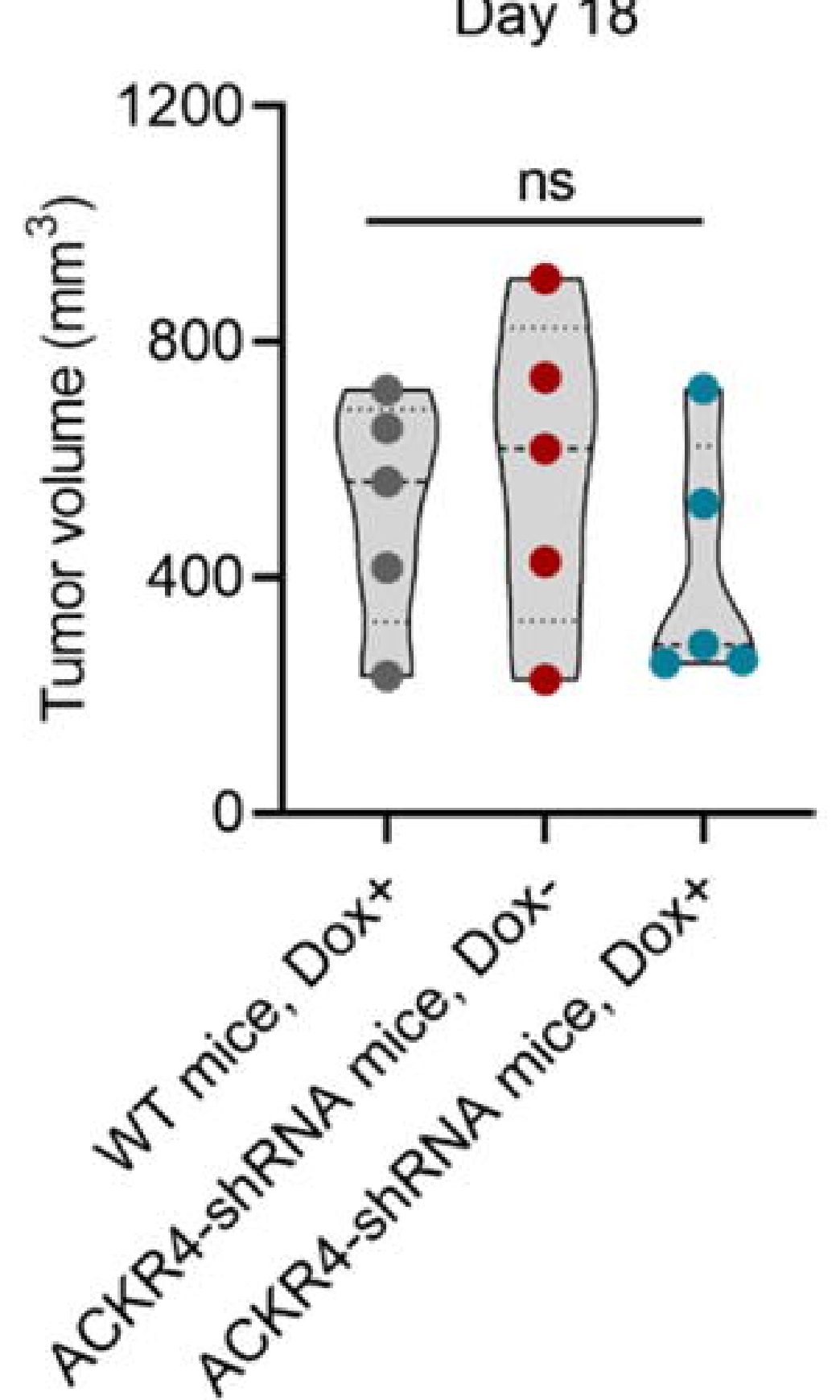


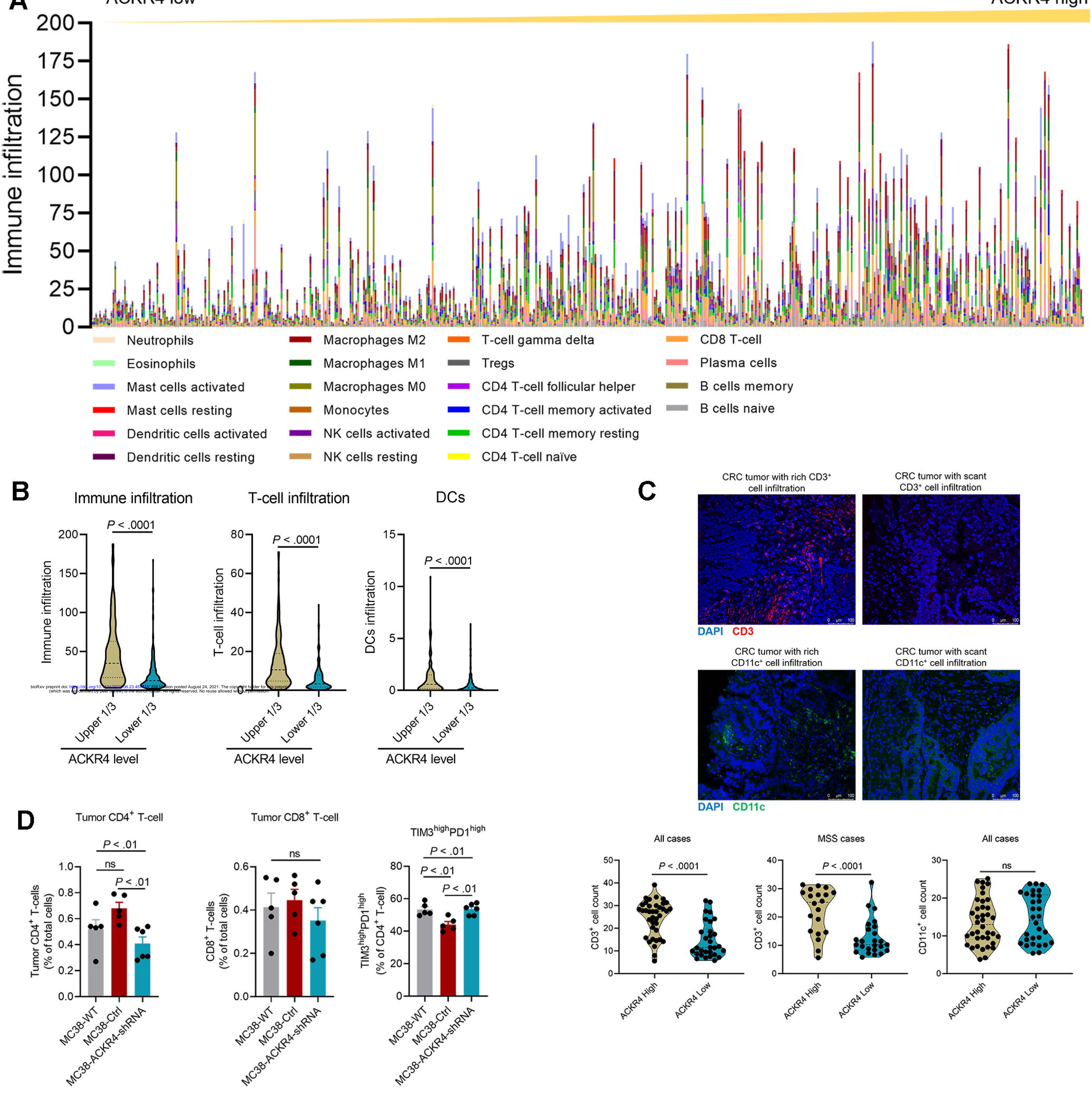




\section{A}
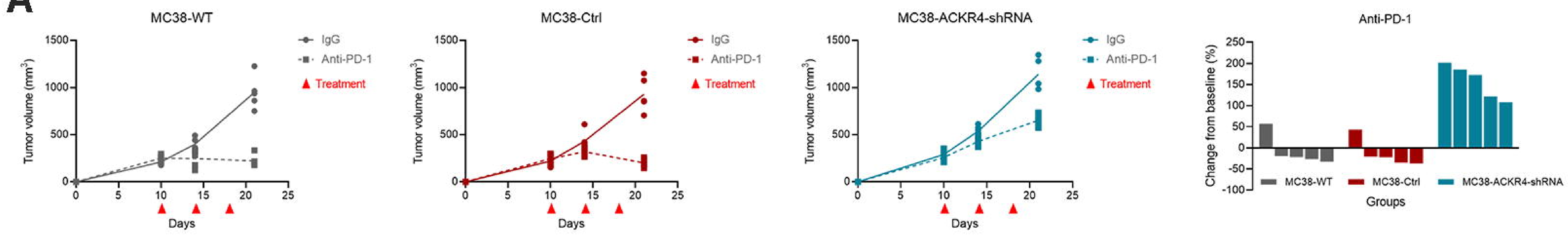

\section{B}

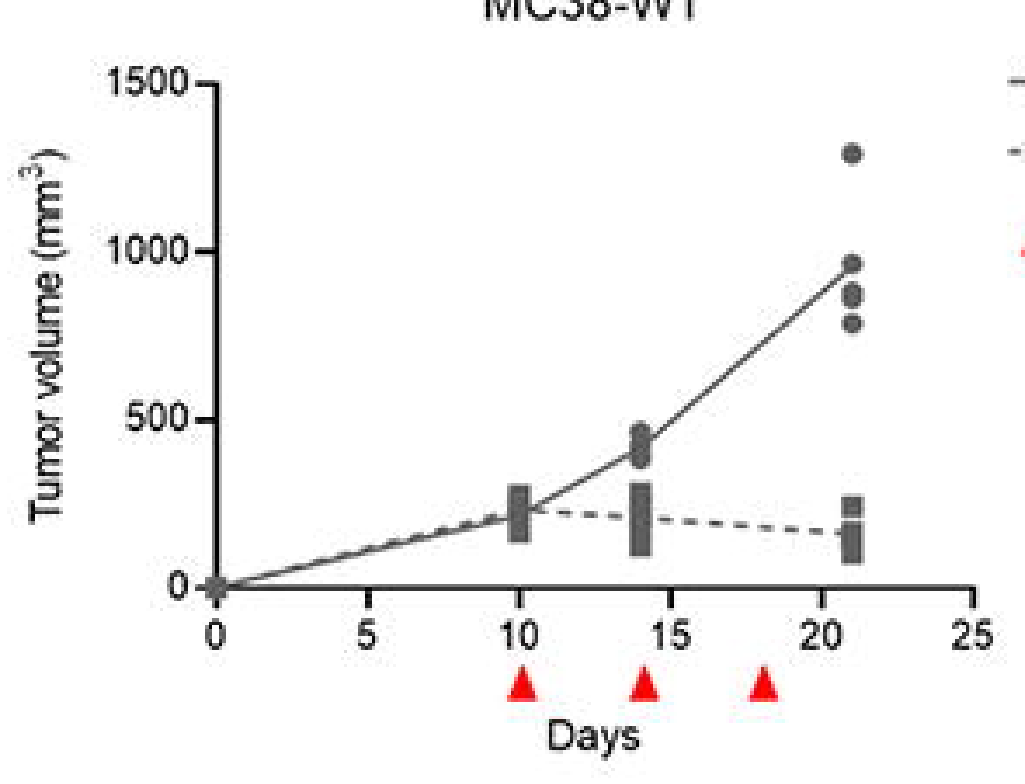

MC38-CtrI

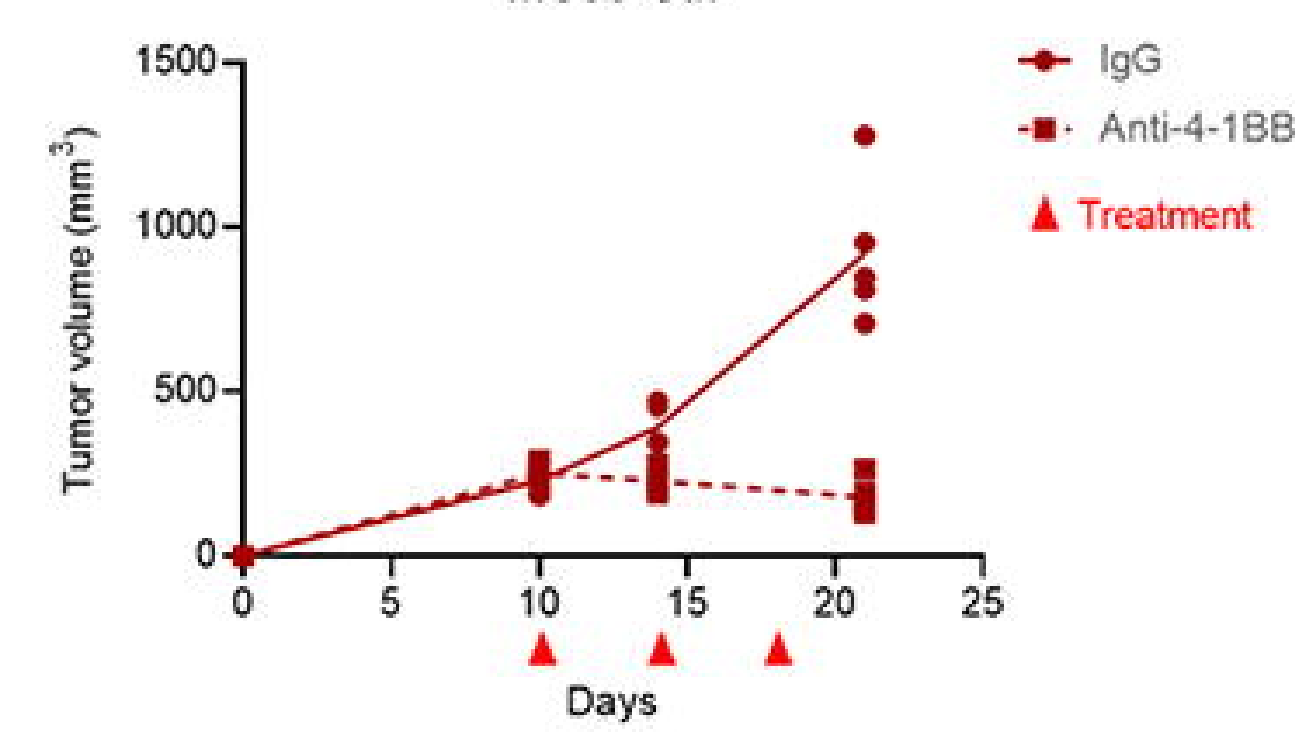

MC38-ACKR4-shRNA

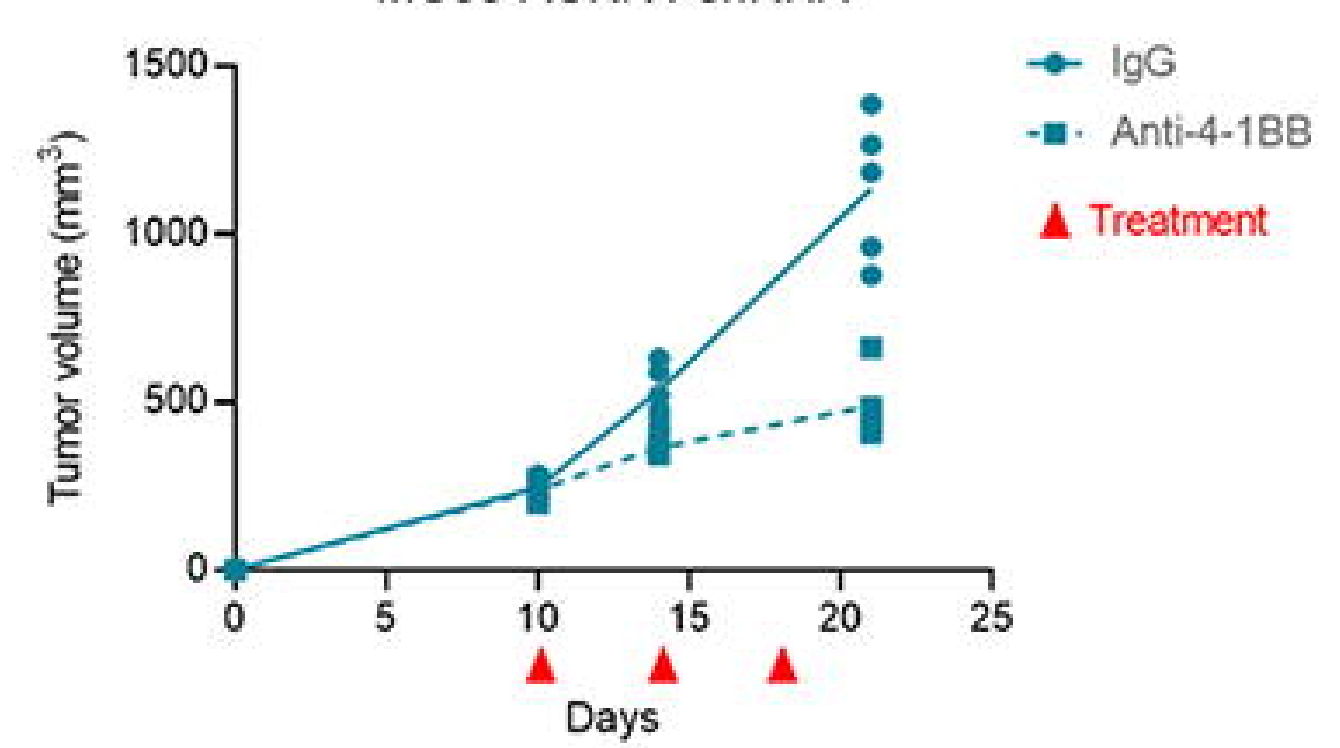

Anti-4-1BB

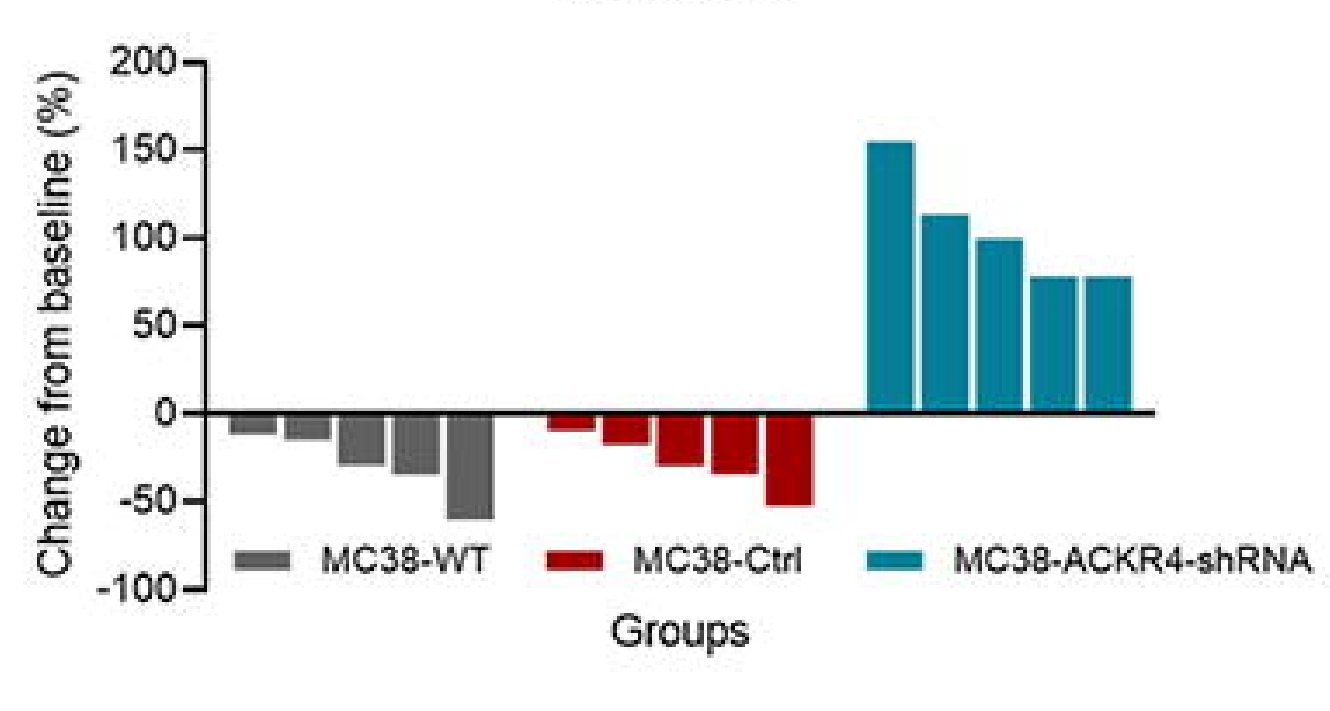


A HuACKR4 3'UTR $\quad 5^{\prime}$...UUCAAAAUAAAACAAGUUAAAAA... Has-miR-552-5p 3' GGUUGUCCGUUUUCCAAUUUG

B

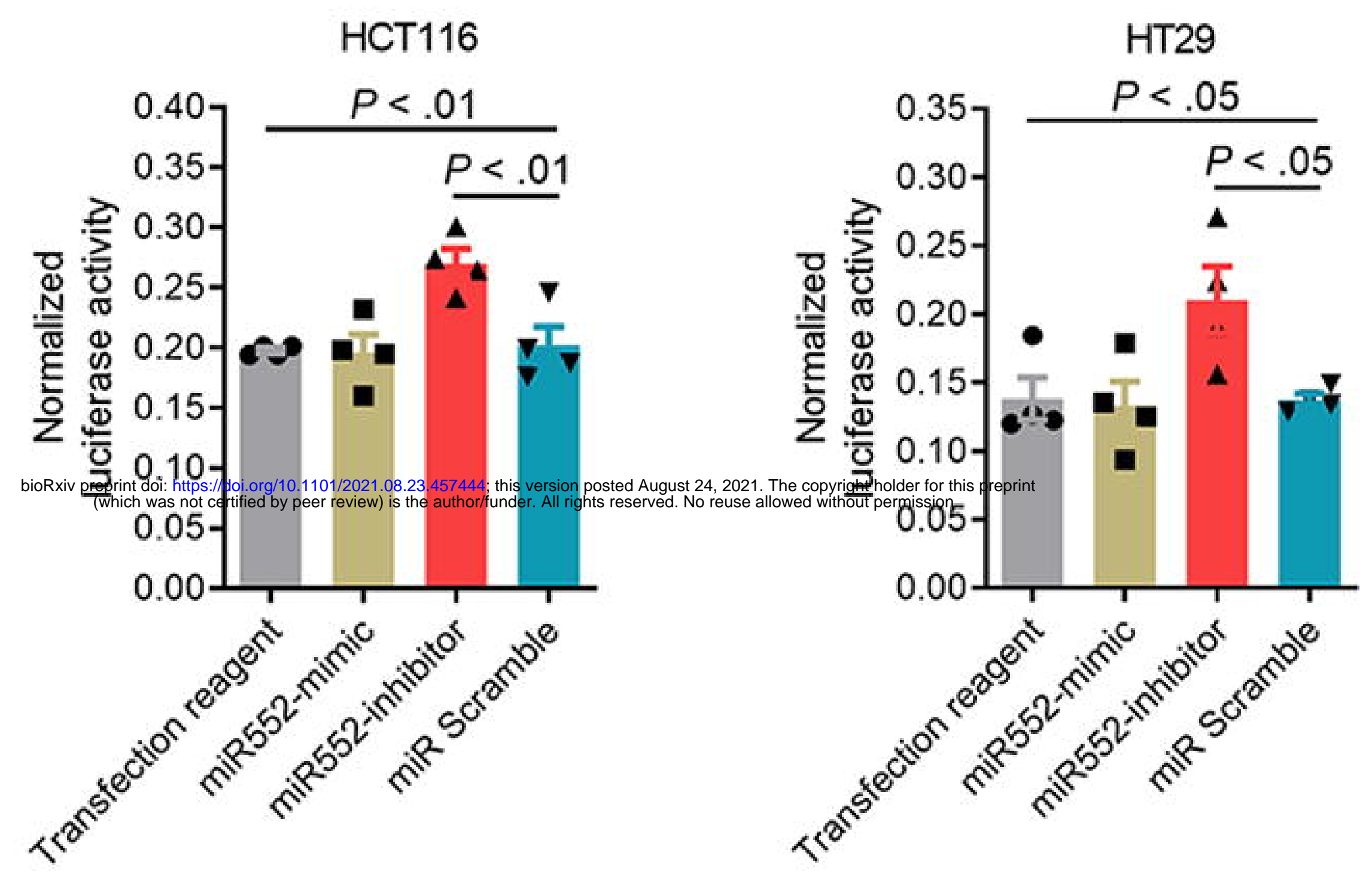

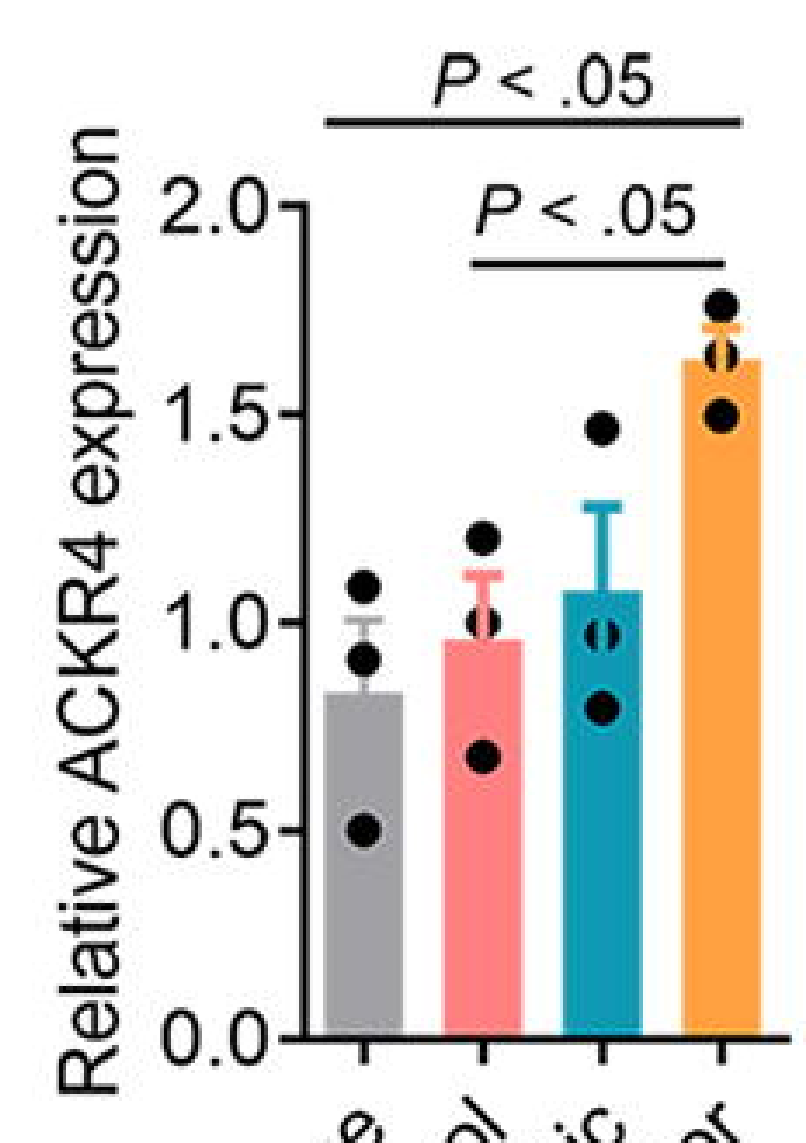

D

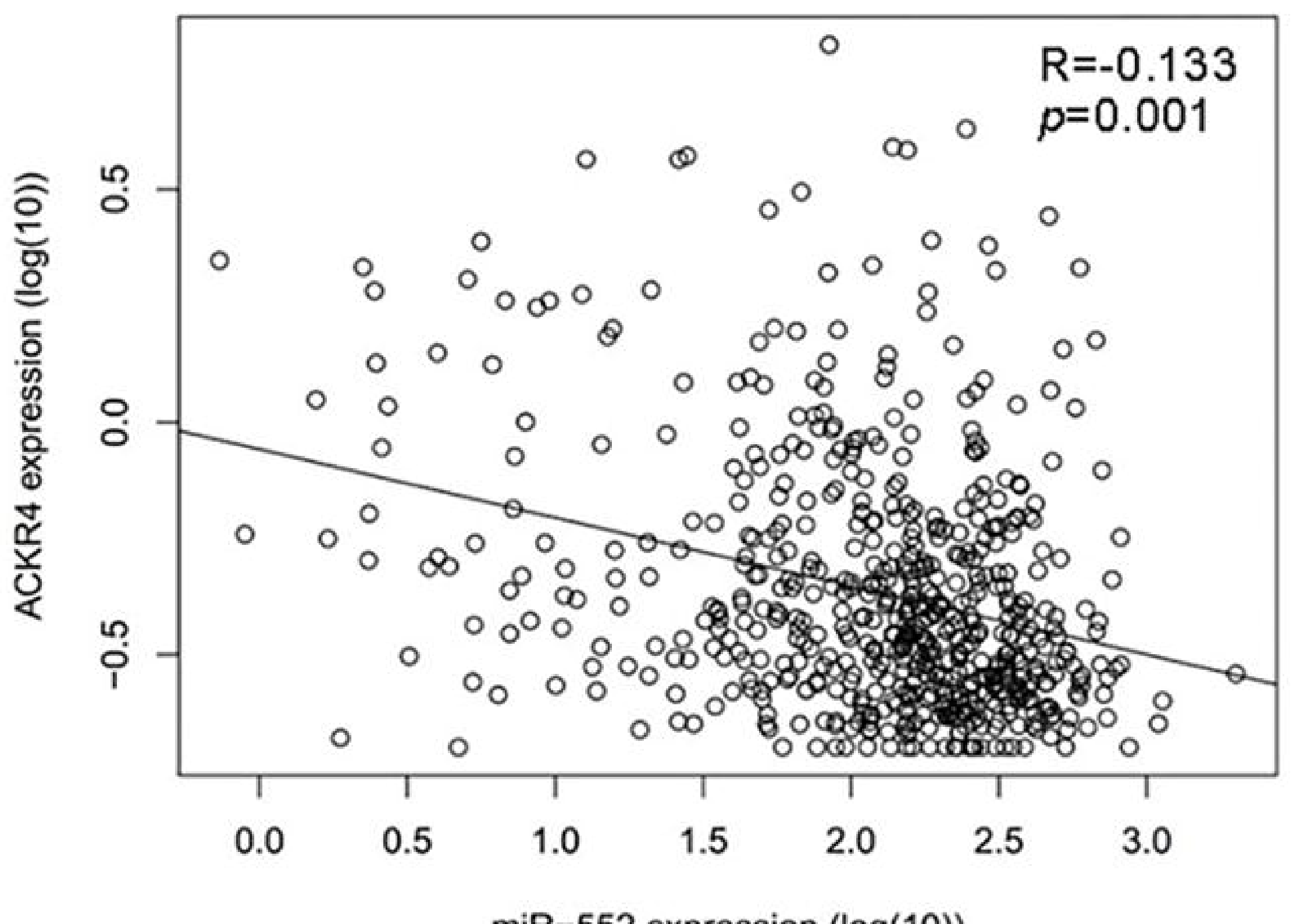

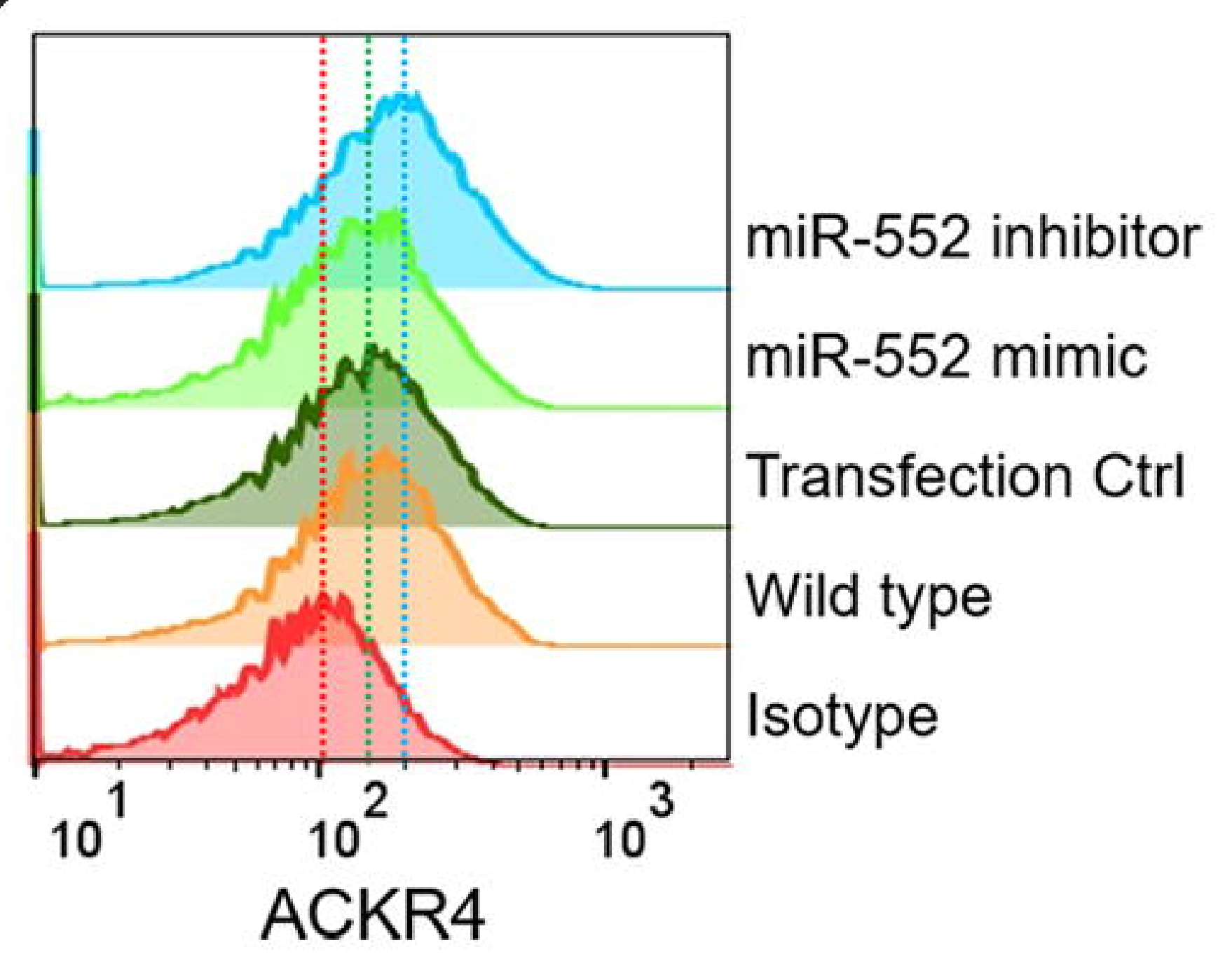

e 0 o

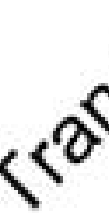

miR-552 expression $(\log (10))$

otal prote
stain

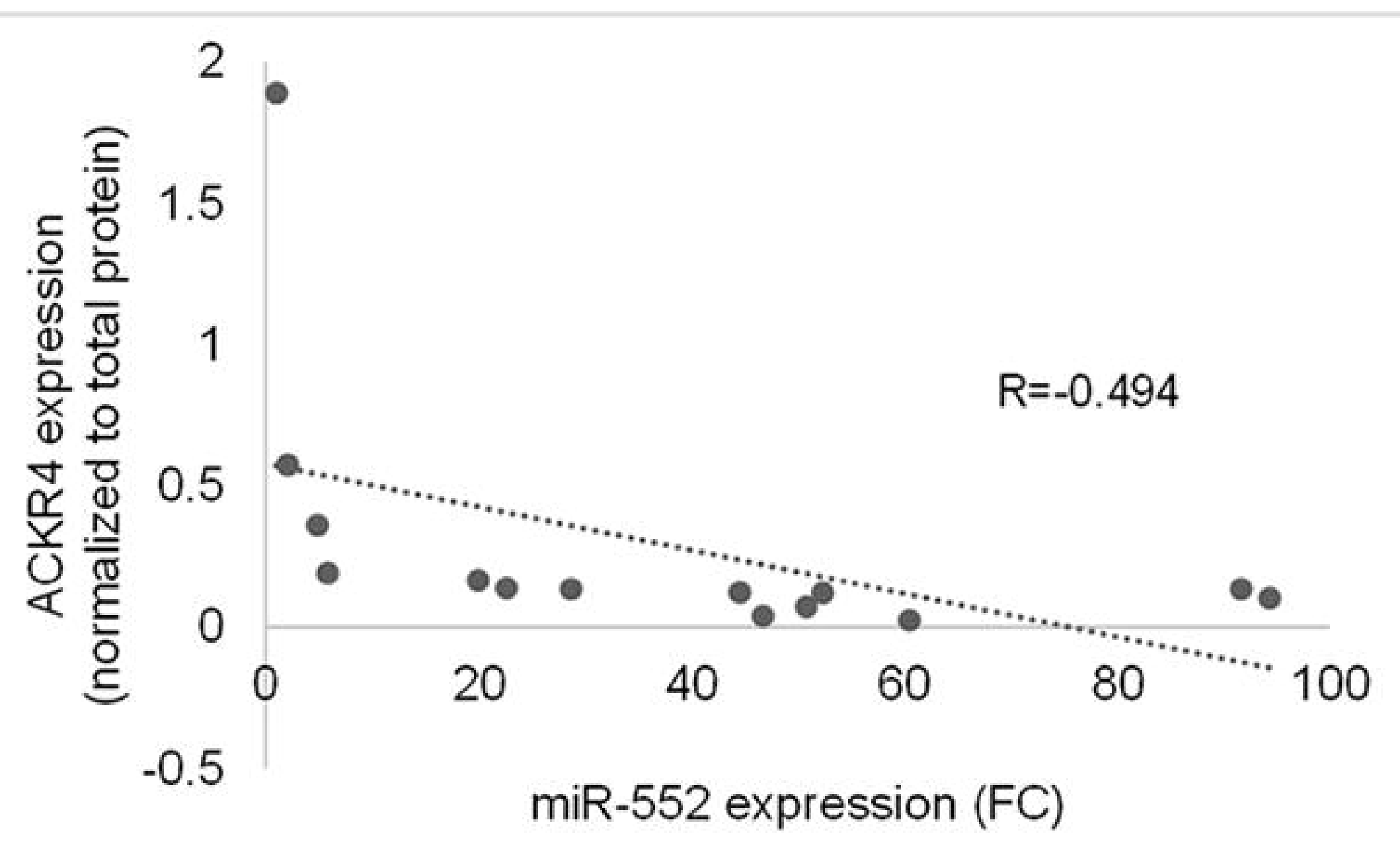

Cumor case:\#1-\#14

$$
\text { ACKR4 }
$$




\section{Lymph Node (LN)}

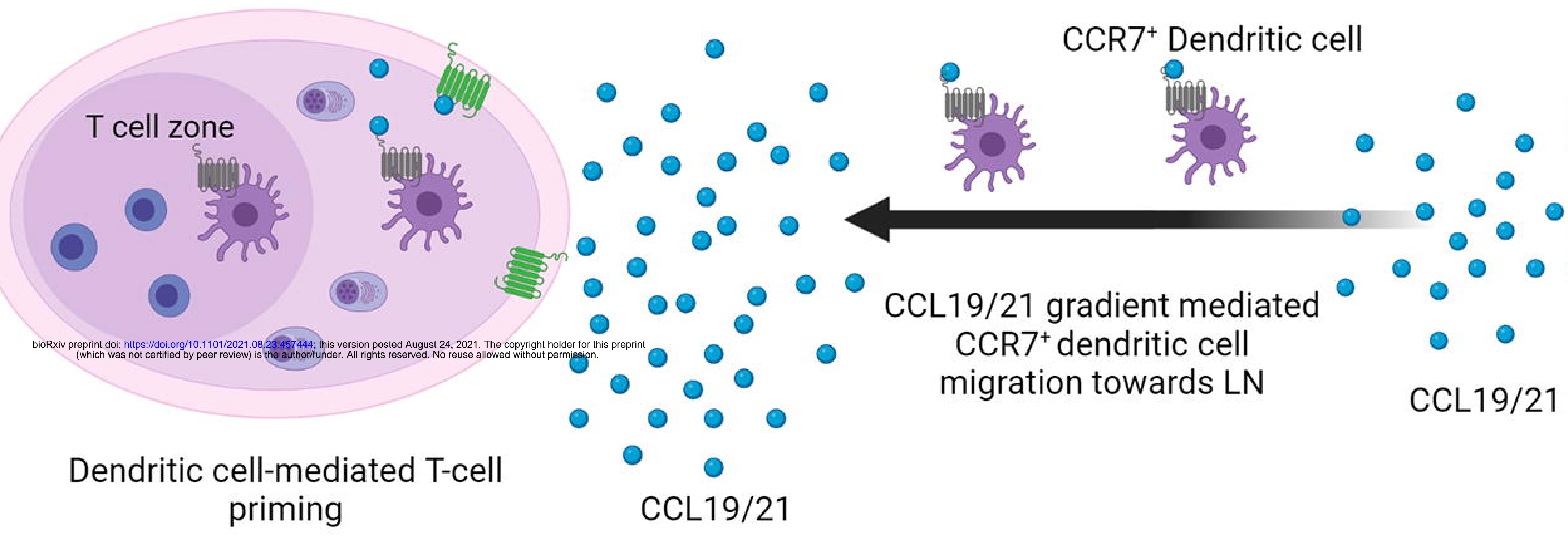

Normal tissue

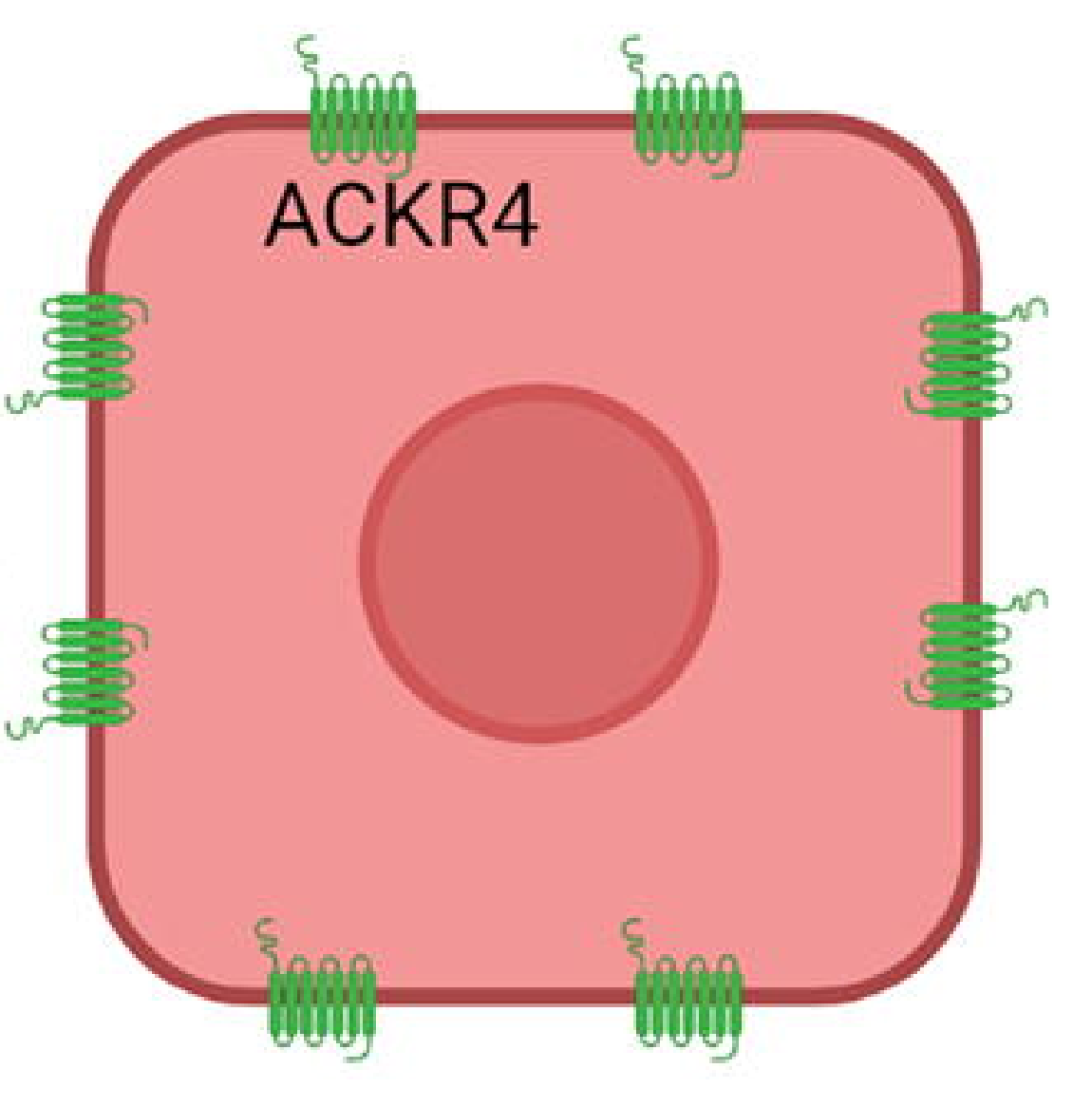

Normal colon tissue with high level of ACKR4

\section{Lymph Node (LN)}

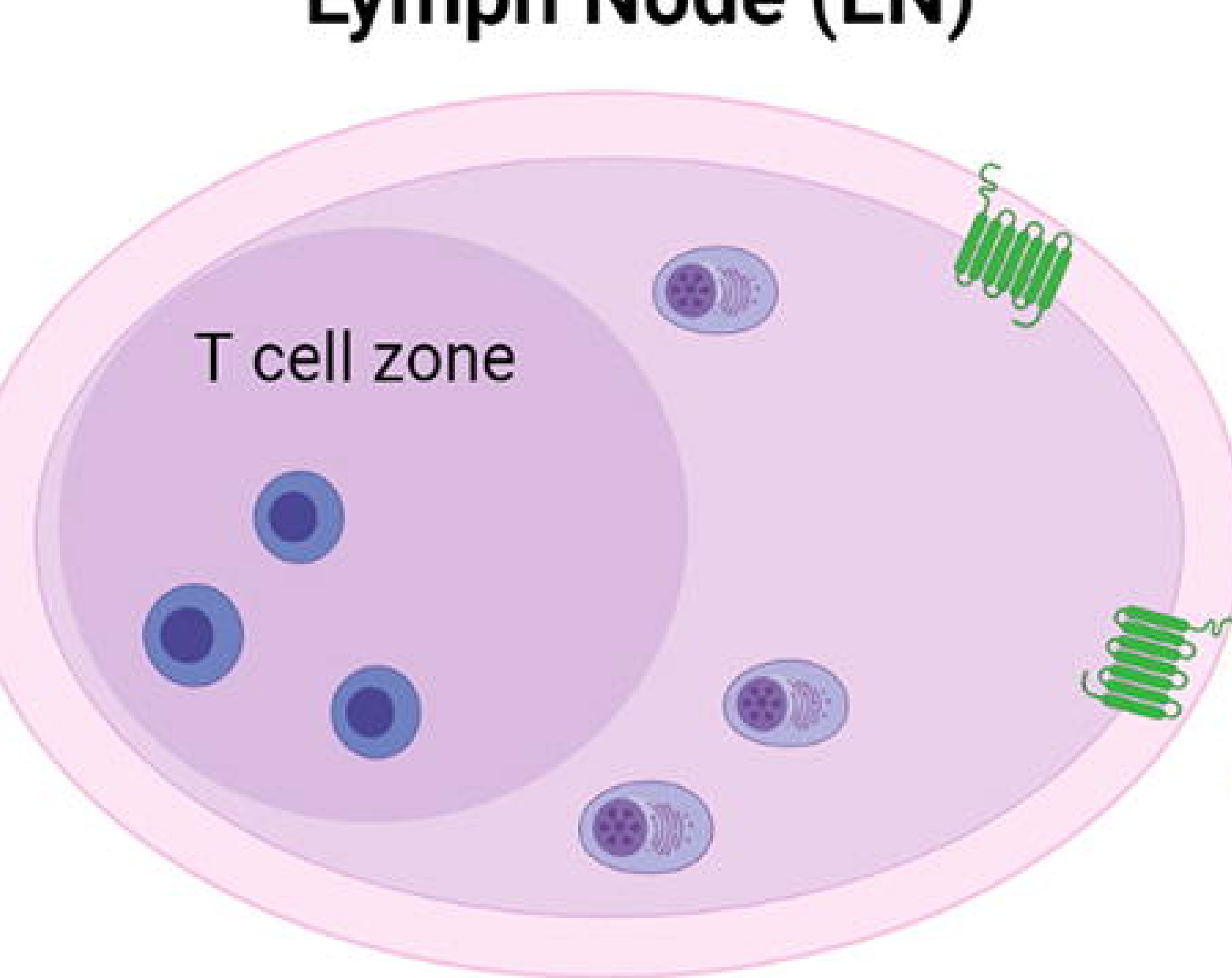

No Dendritic cell-mediated T-cell priming

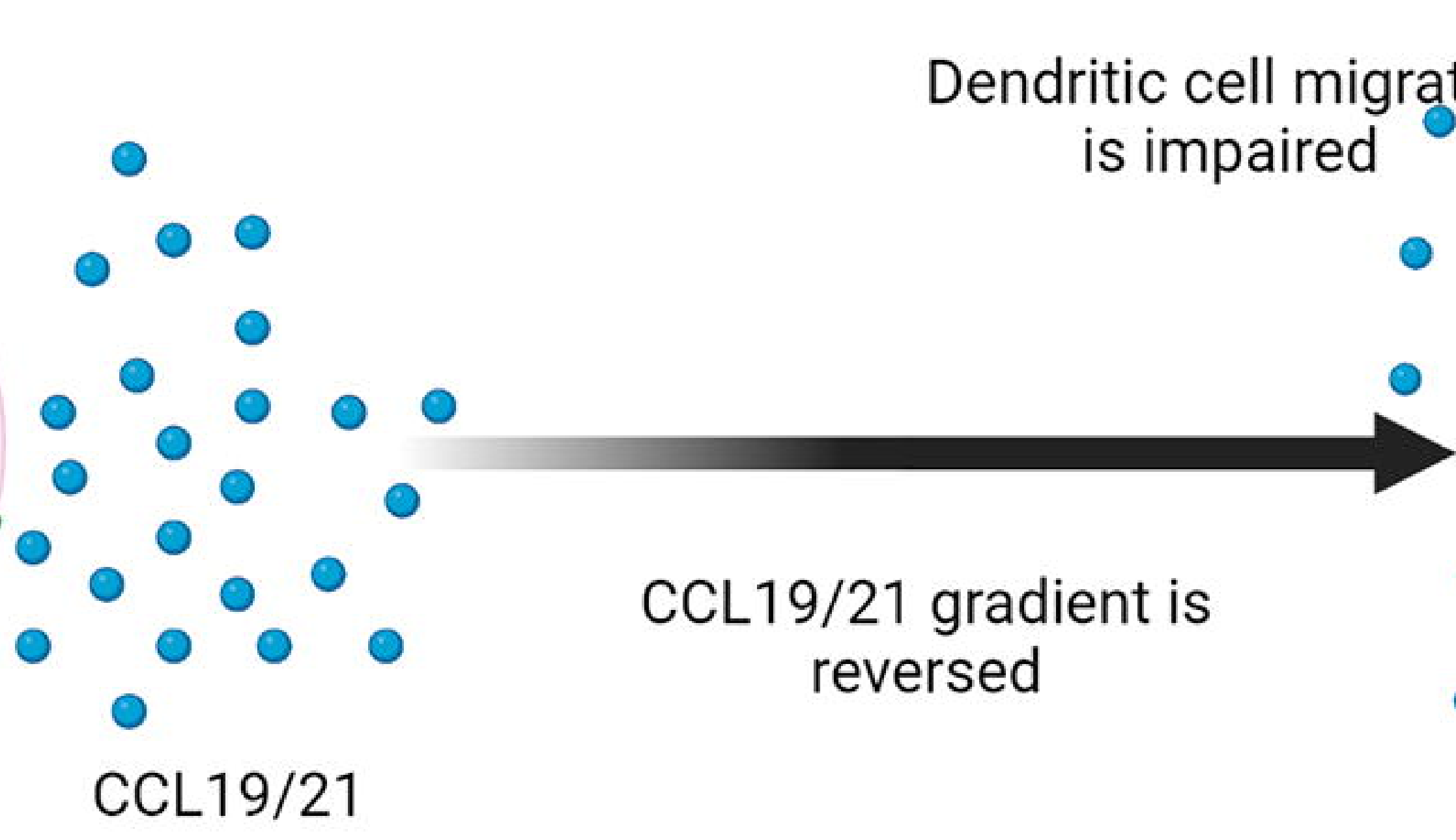

Accumulation of CCL19/21

\section{Tumor tissue}

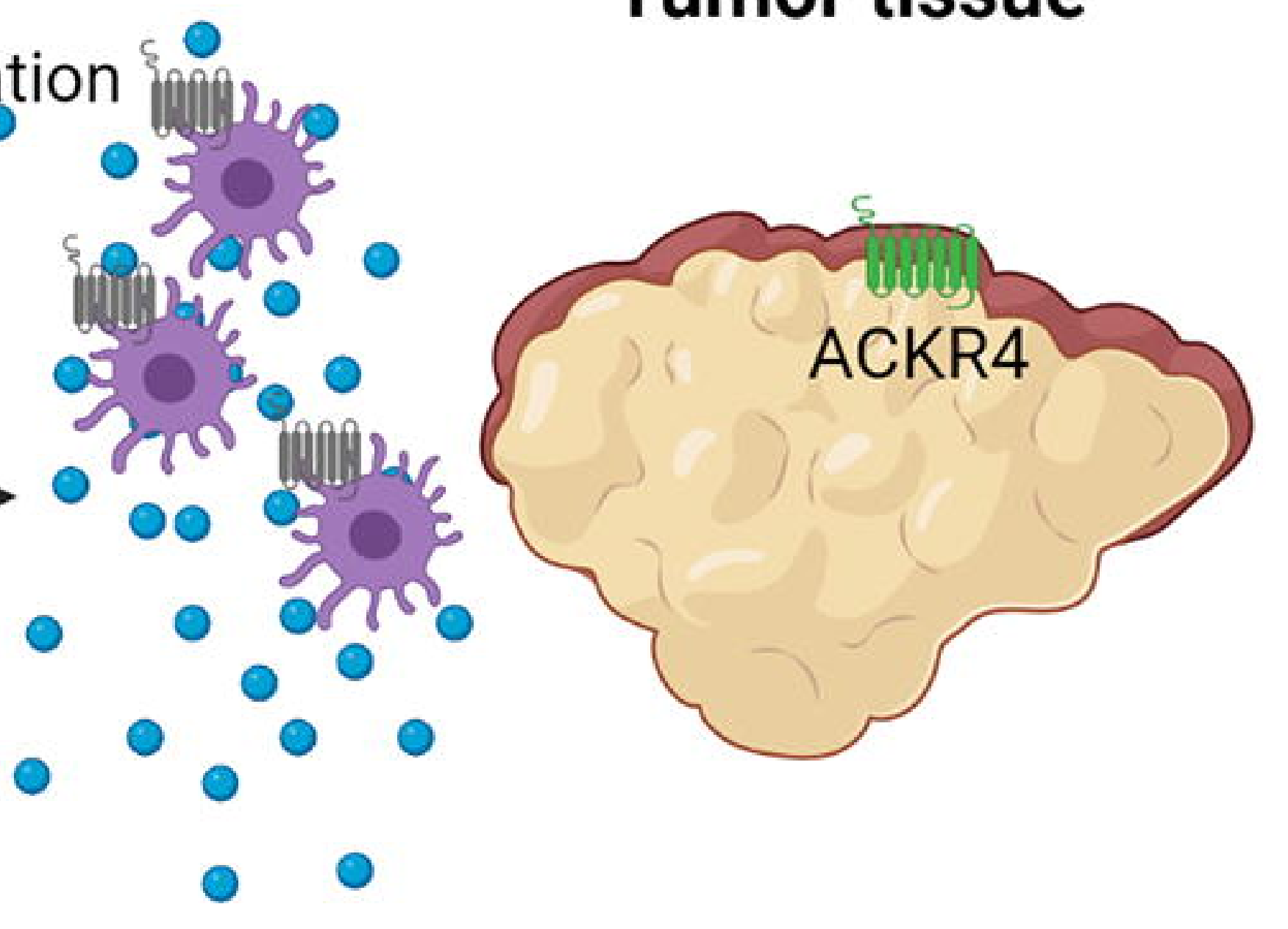

Tumor tissue with low level of ACKR4 


\section{A}

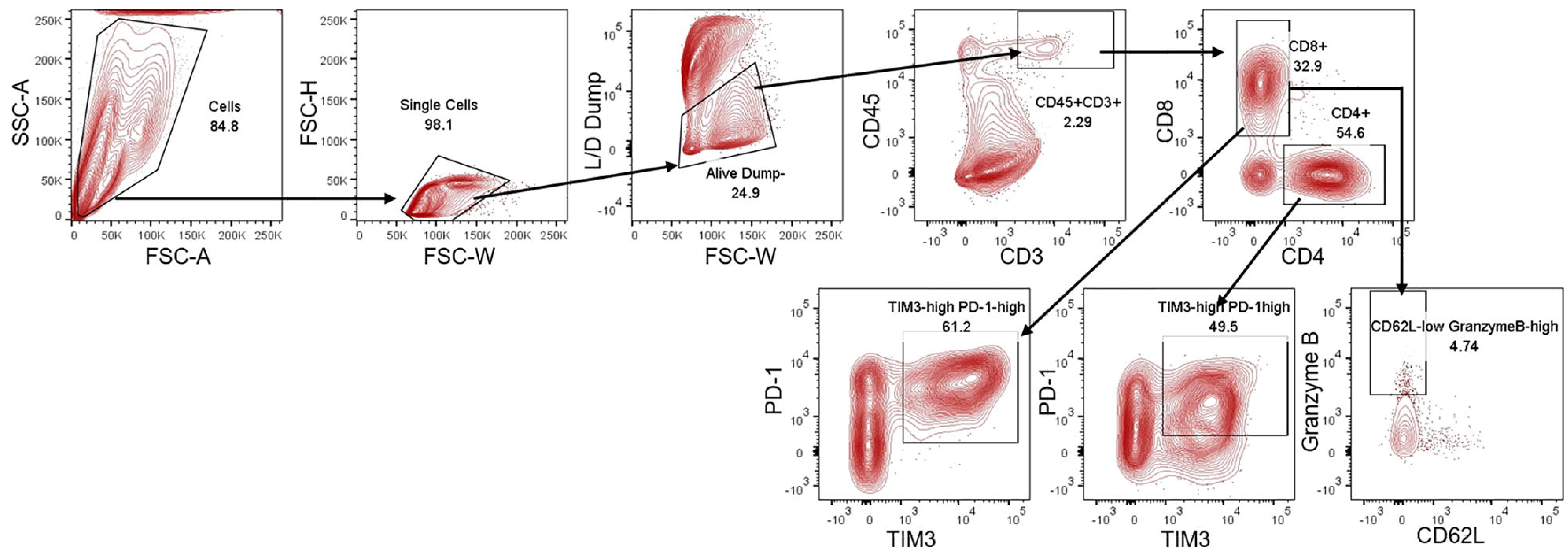

B
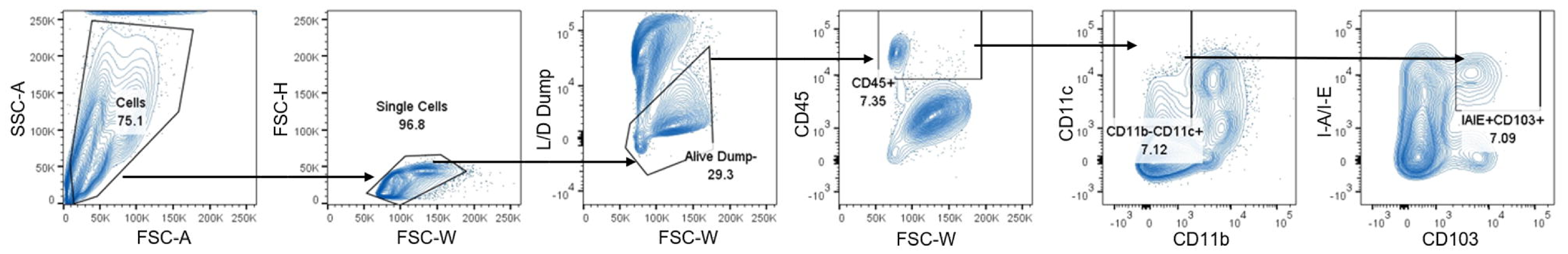

C ${ }_{\mathrm{TIM}} 3^{\text {high }}{ }_{\mathrm{PD}} 1^{\text {high }}$

CD62L ${ }^{\text {low }}$ GranzymeB ${ }^{\text {high }}$

Tumor CD103 ${ }^{+}$DCs

$\mathrm{CD} 80$ on $\mathrm{CD}_{103^{+}} \mathrm{DCs}$

CD86 on $\mathrm{CD}_{103^{+}} \mathrm{DCs}$
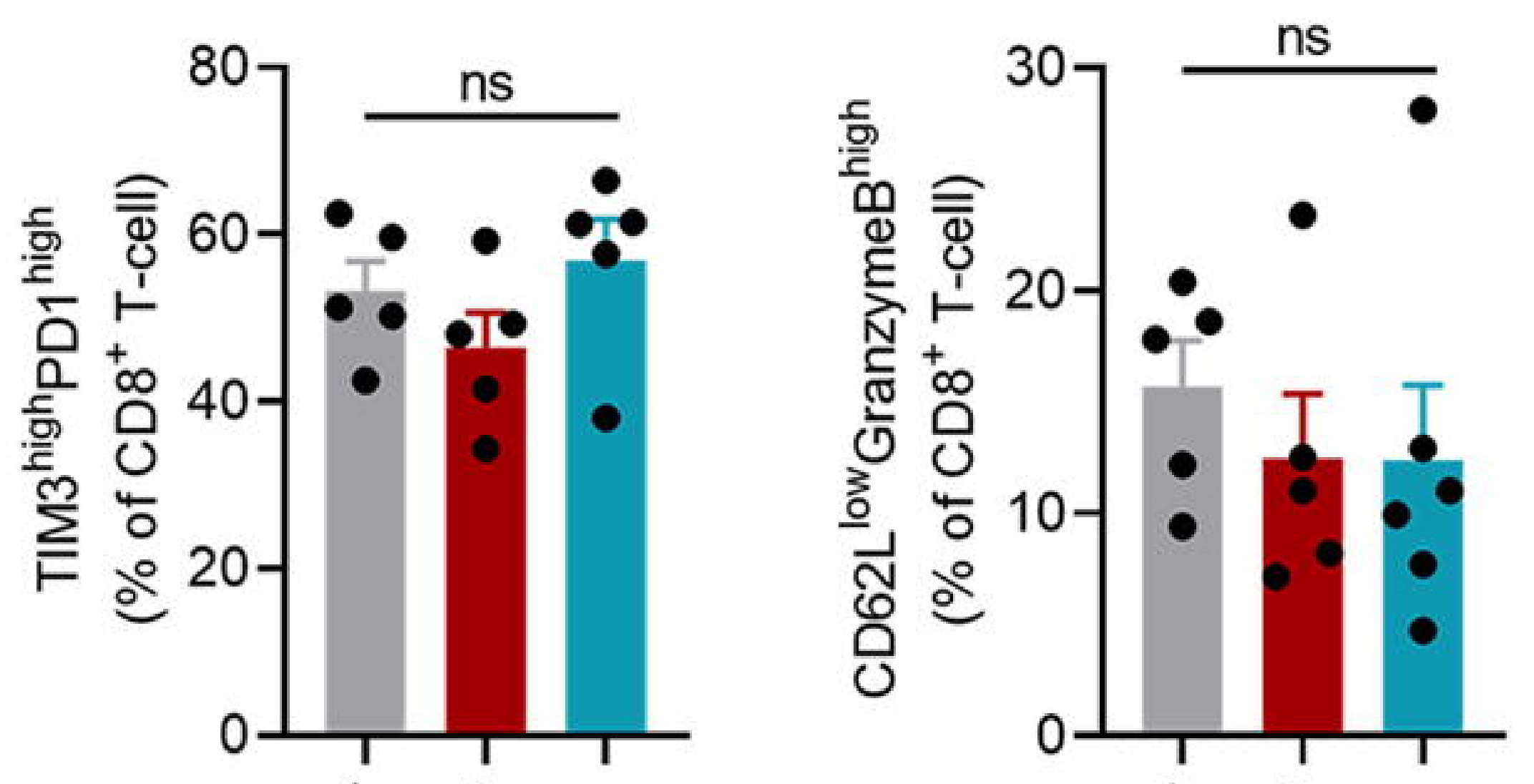

D

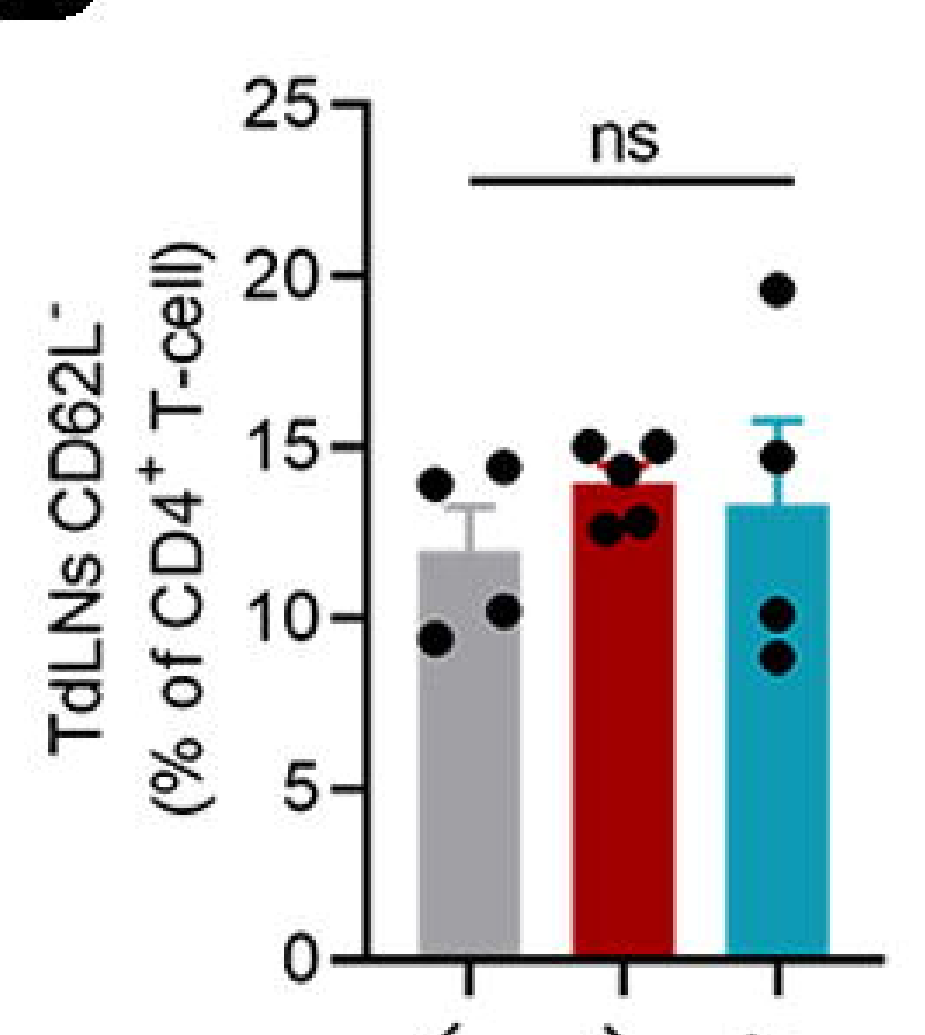

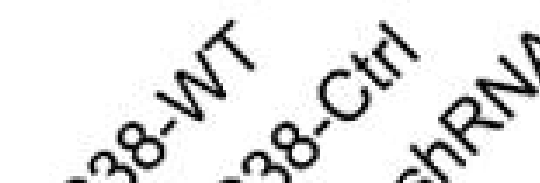
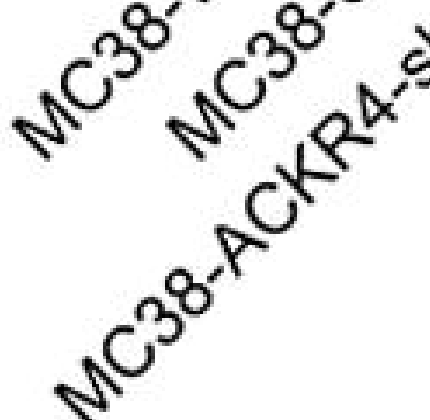
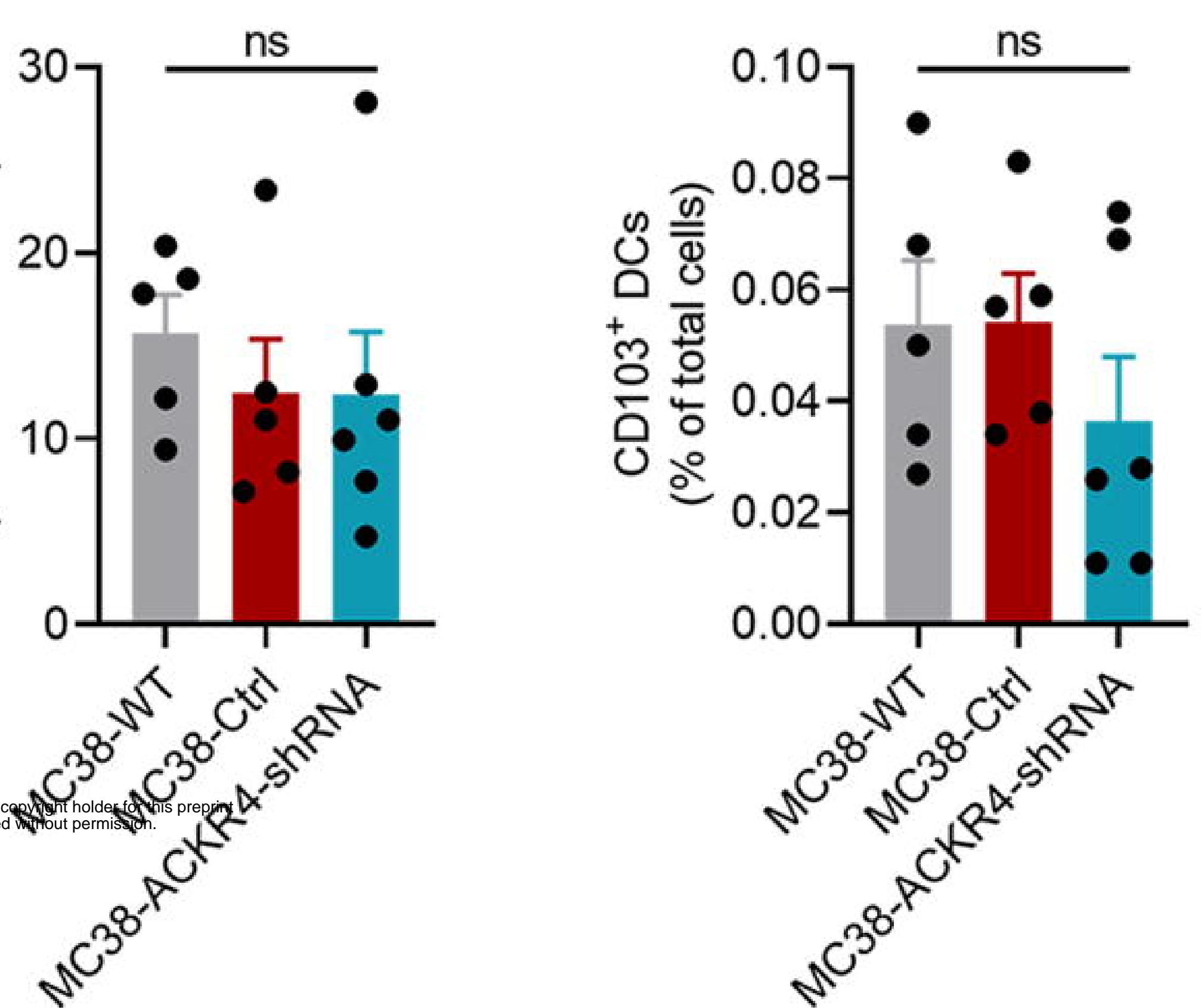

TdLNs CD62L ${ }^{-C D 8}{ }^{+} \mathrm{T}$-cell
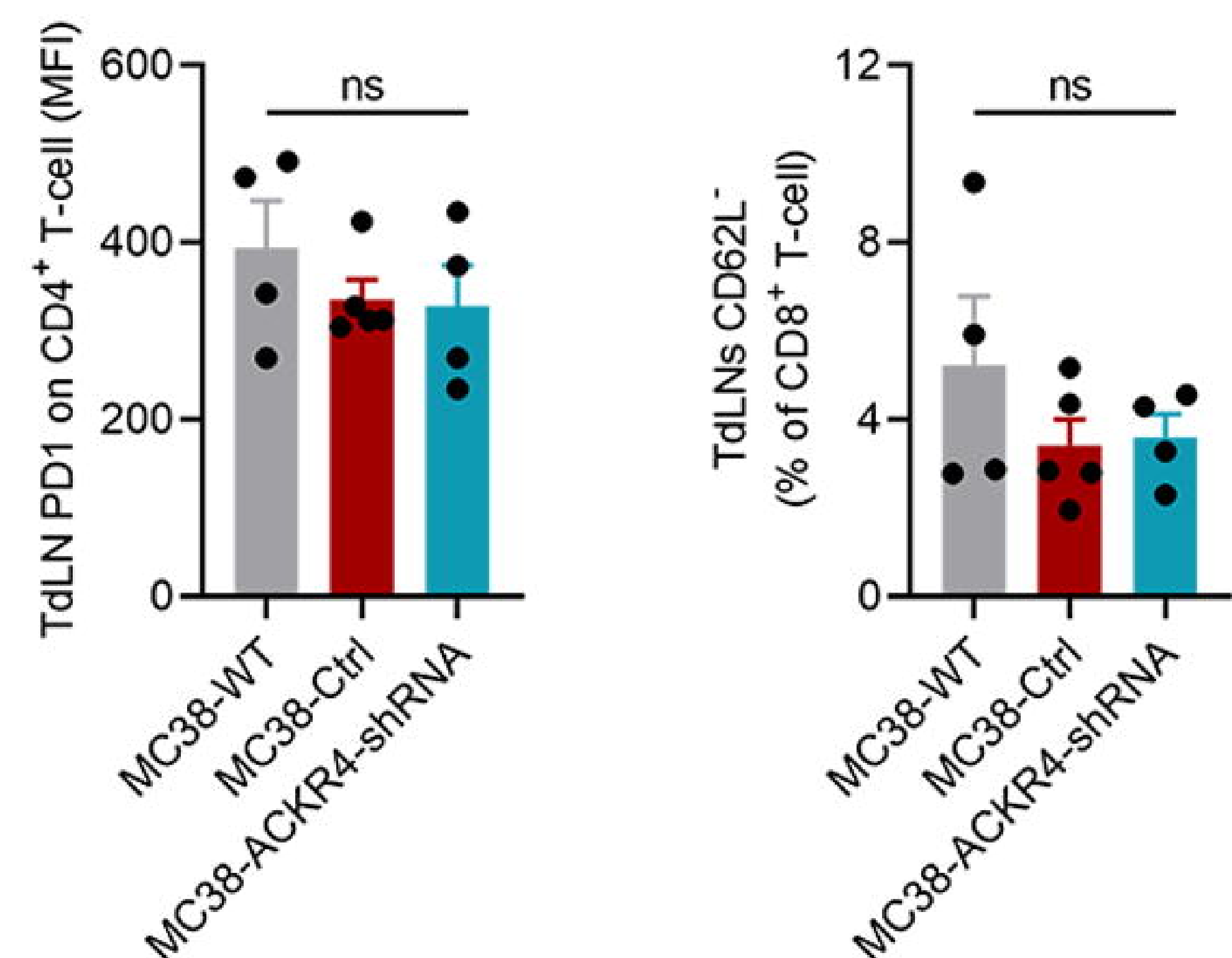
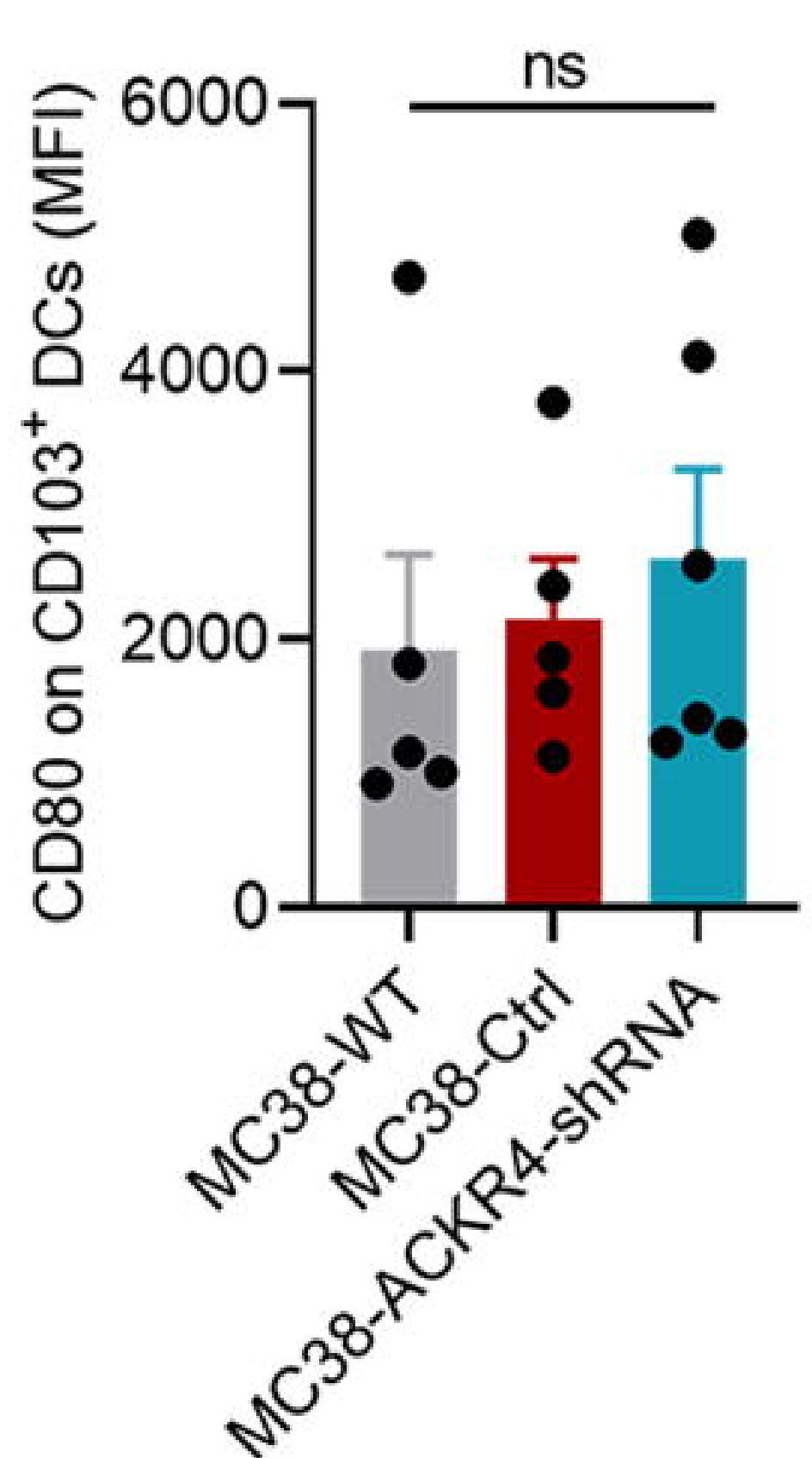

TdLN PD1 on $\mathrm{CD}^{+} \mathrm{T}$-cell
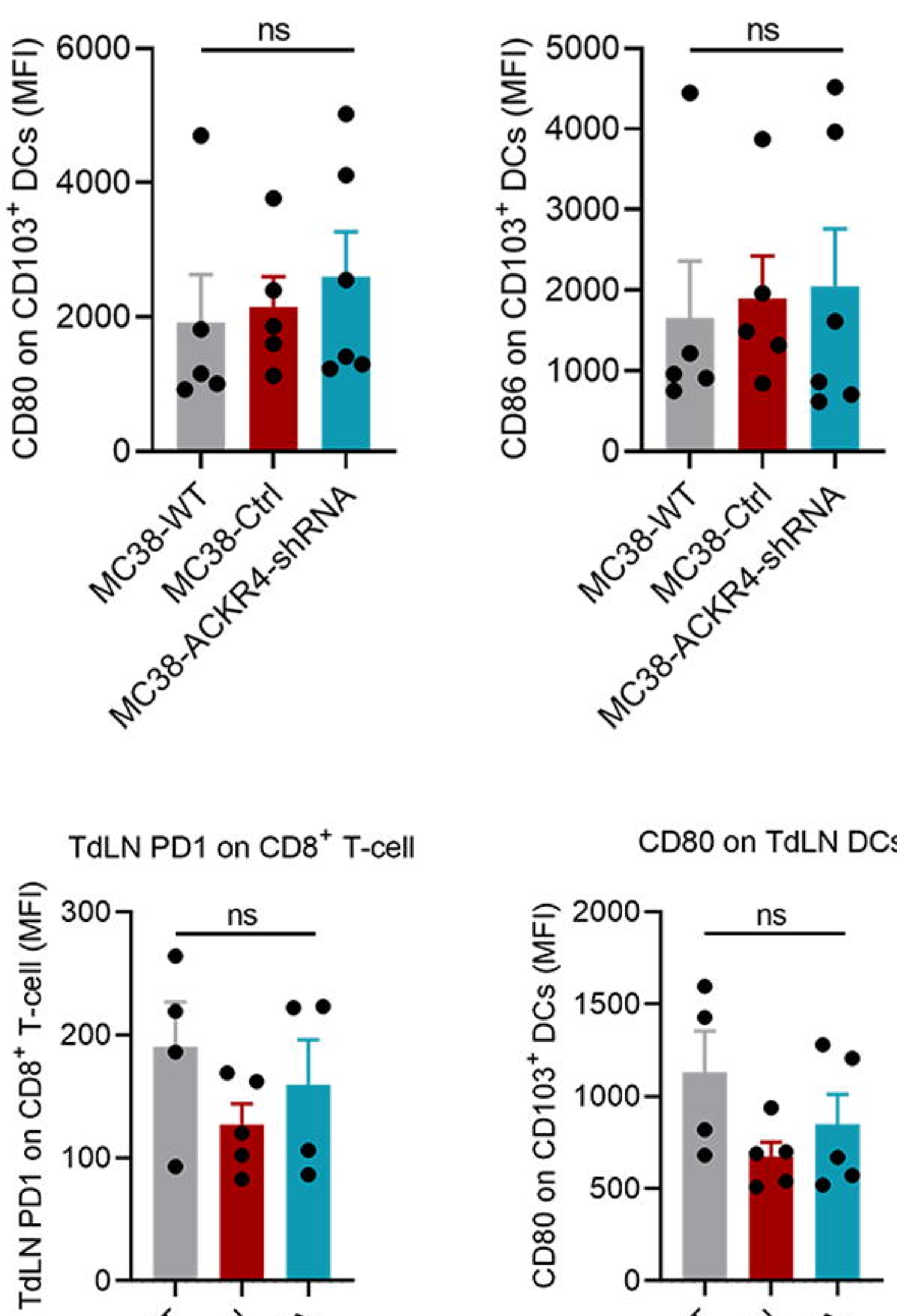

CD80 on TdLN DCs
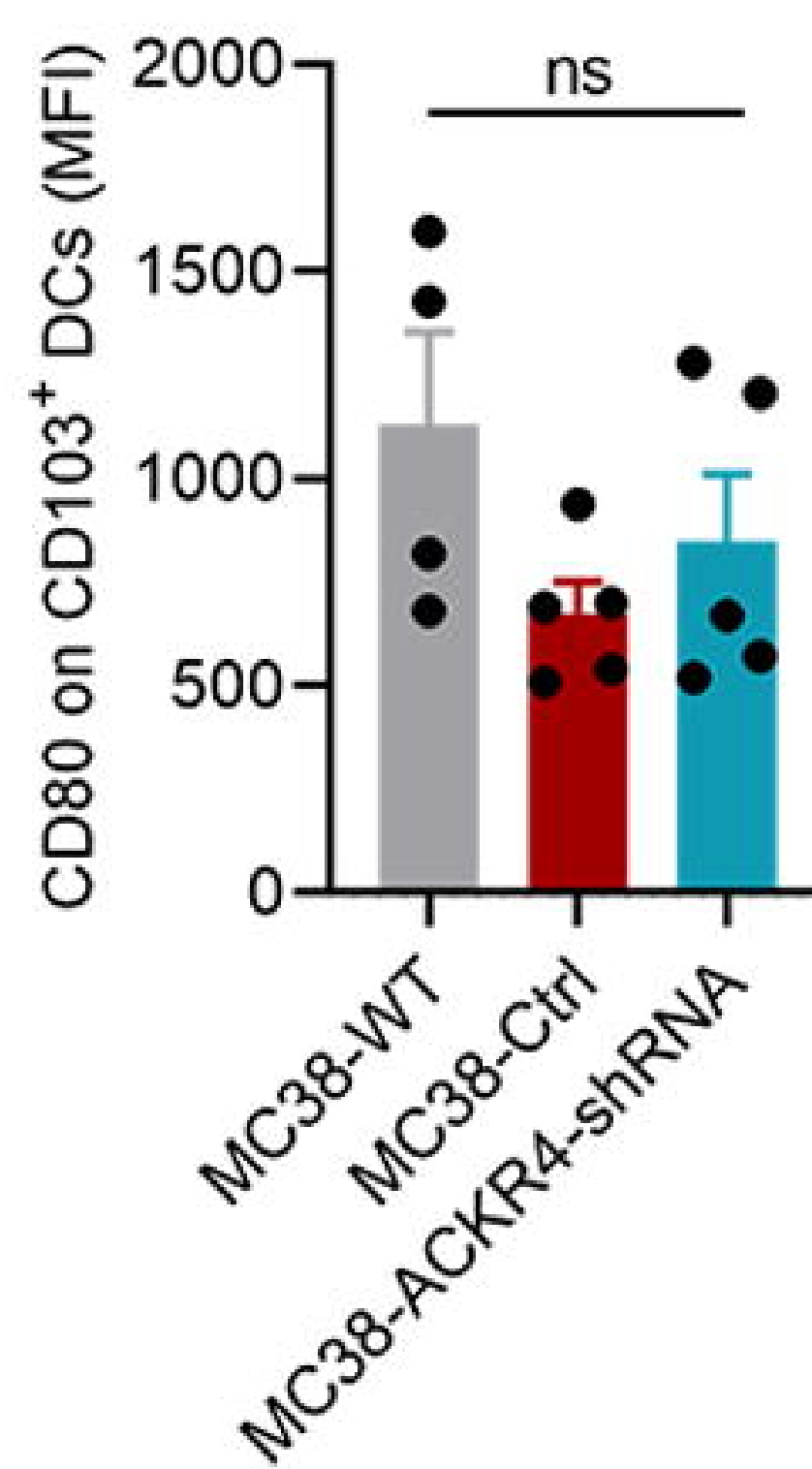

CD86 on TdLN DCs
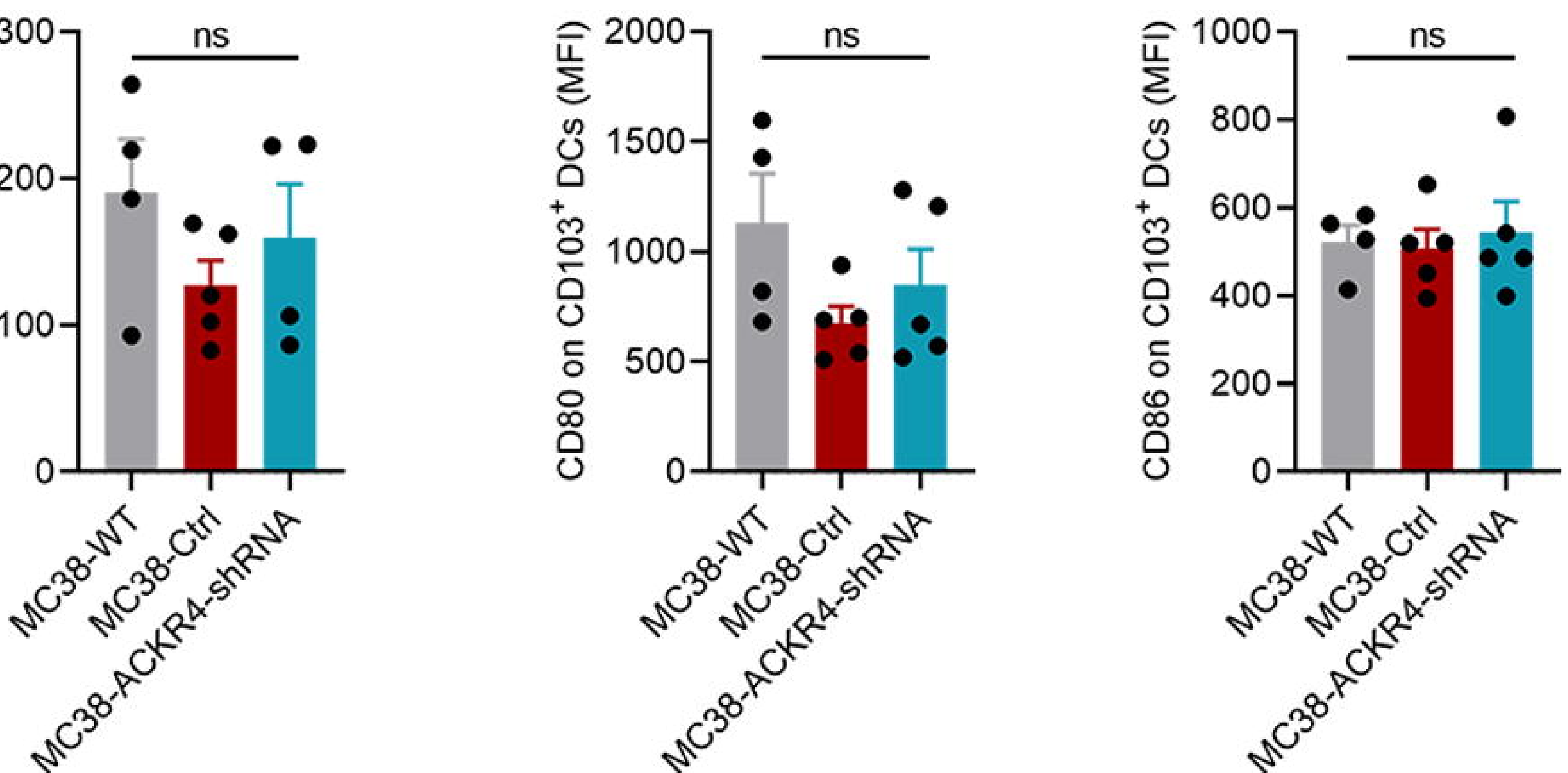

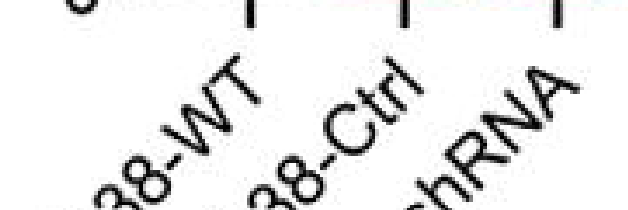

